Comment. Math. Helv. 73 (1998) 603-636

0010-2571/98/040603-34\$1.50+0.20/0
(C) 1998 Birkhäuser Verlag, Basel

Commentarii Mathematici Helvetici

\title{
Schubert polynomials and Bott-Samelson varieties
}

\author{
Peter Magyar*
}

\begin{abstract}
Schubert polynomials generalize Schur polynomials, but it is not clear how to generalize several classical formulas: the Weyl character formula, the Demazure character formula, and the generating series of semistandard tableaux. We produce these missing formulas and obtain several surprising expressions for Schubert polynomials.

The above results arise naturally from a new geometric model of Schubert polynomials in terms of Bott-Samelson varieties. Our analysis includes a new, explicit construction for a BottSamelson variety $Z$ as the closure of a $B$-orbit in a product of flag varieties. This construction works for an arbitrary reductive group $G$, and for $G=G L(n)$ it realizes $Z$ as the representations of a certain partially ordered set.

This poset unifies several well-known combinatorial structures: generalized Young diagrams with their associated Schur modules; reduced decompositions of permutations; and the chamber sets of Berenstein-Fomin-Zelevinsky, which are crucial in the combinatorics of canonical bases and matrix factorizations. On the other hand, our embedding of $Z$ gives an elementary construction of its coordinate ring, and allows us to specify a basis indexed by tableaux.
\end{abstract}

Mathematics Subject Classification (1991). 14M15, 16G20.

Keywords. Flag variety, Young tableaux, Demazure character formula.

\section{Introduction}

The classical Schur polynomials appear in many contexts: 1) as characters of the irreducible representations of $G L(n, \mathbf{C})$ (the Schur modules); 2) as an algebraic model for the cohomology ring of a Grassmannian (product of Schur polynomials $\leftrightarrow$ intersection of Schubert classes); 3) as an orthogonal basis for the symmetric functions in a polynomial ring; and 4) as generating functions enumerating semistandard Young tableaux. (See [10] for a unified account of this theory.)

In recent decades many generalizations of Schur polynomials have appeared, among the most interesting being the Schubert polynomials first defined by Lascoux and Schutzenberger [15]. These are known to generalize each of the above interpretations. They are: 1) characters of representations of the group $B$ of upper

*Partially supported by the National Science Foundation. 
triangular matrices [12]; 2) an algebraic model of the cohomology ring of a flag variety [4], [7]; 3) an orthogonal basis for a polynomial ring [15]; and 4) generating functions for certain mysterious tableaux defined by compatiblity conditions in the plactic monoid [16].

Nevertheless, many of the rich properties of Schur polynomials have no known analogs for Schubert polynomials. In this paper we supply several such missing analogs, mainly concerning interpretations 1) and 4): analogs of the Weyl and Demazure character formulas; and a straightforward construction for the mysterious tableaux of Lascoux and Schutzenberger, showing how they "quantize" our Demazure formula. These results also hold for a broad class of Schur-like polynomials associated to generalized Young diagrams, such as skew Schur polynomials [1], [23], [24], [25], [21].

These results are purely combinatorial, but we obtain them by generalizing a powerful tool of representation theory, the Borel-Weil Theorem, which states that Schur modules (whose characters are Schur polynomials) are graded pieces in the coordinate ring of a flag variety (c.f. [10]). The theory of Schur polynomials can be developed from this point of view, and this is what we do for Schubert polynomials and their associated $B$-modules. Instead of flag varieties, however, we must use the more general varieties defined by Bott and Samelson, which are a well-known tool in geometric representation theory. (They are indexed by reduced decompositions of permutations into simple transpositions.)

This method follows our paper [21], but we must do extra geometric work here, giving a precise connection between our $B$-modules and the Bott-Samelson varieties $Z$. As a by-product of our analysis, we obtain a new construction of the BottSamelson varieties for an arbitrary reductive group $G$. In our case $G=G L(n)$, the new construction realizes $Z$ as the variety of representations of a partially ordered set. This poset is equivalent to two well-known but previously unconnected combinatorial pictures, and our approach reveals deep relations between them: first, generalized Young diagrams, which are used to construct generalized Schur modules; and second, reduced decompositions of permutations, which are pictured via the wiring diagrams and chamber sets of Berenstein, Fomin, and Zelevinsky [2], [18], crucial in the combinatorics of matrix factorizations, total positivity, and canonical bases.

The paper is organized into three parts, which may be read independently and have separate introductions. The first part $(\S 1)$ introduces Bott-Samelson varieties for a general reductive group, and shows the isomorphism between our new construction and the classical one. This lays the groundwork for our papers [13], [14] with V. Lakshmibai, giving a Standard Monomial Theory for Bott-Samelson varieties.

The second part ( $\S 2-3)$ makes this construction explicit for $G L(n)$, discusses the combinatorial models and their connections, defines generalized Schur modules and Schur polynomials, and proves the Demazure character formula for them.

The last part $(\S 4)$ states all the applications to Schubert polynomials in elementary combinatorial language. 


\section{Bott-Samelson varieties}

Let $G$ be a complex reductive Lie group (or more generally a reductive algebraic group over an infinite field of arbitrary characteristic or over $\mathbf{Z}$ ), and let $B$ be a Borel subgroup.

The Bott-Samelson varieties are an important tool in the representation theory of $G$ and the geometry of the flag variety $G / B$. First defined in [5] as a desingularization of the Schubert varieties in $G / B$, they were exploited by Demazure [7] to analyze the singular cohomology or Chow ring $H^{*}(G / B, \mathbf{C})$ (the Schubert calculus), and the projective coordinate ring $\mathbf{C}[G / B]$. Since the irreducible representations of $G$ are embedded in the coordinate ring, Demazure was able to obtain an iterative character formula [8] for these representations.

Bott-Samelson varieties are so useful because they "factor" the flag variety into a "product" of projective lines. More precisely, they are iterated $\mathbf{P}^{1}$-fibrations and they each have a natural, birational map to $G / B$. The Schubert subvarieties themselves lift birationally to iterated $\mathbf{P}^{1}$-fibrations under this map (hence the desingularization). The combinatorics of Weyl groups enters because a given $G / B$ can be "factored" in many ways, indexed by sequences $\mathbf{i}=\left(i_{1}, i_{2}, \ldots, i_{l}\right)$ such that $w_{0}=s_{i_{1}} s_{i_{2}} \cdots s_{i_{l}}$ is a reduced decompostion of the longest Weyl group element $w_{0}$ into simple reflections.

The Bott-Samelson variety $Z_{\mathbf{i}}$ is usually defined as a product of $l$ minimal parabolic subgroups modulo an action of $B^{l}$, but we give a new, dual construction of $Z_{\mathbf{i}}$ as a subvariety rather than a quotient. It is the closure of an orbit of the Borel subgroup $B$ inside a product of flag varieties:

$$
Z_{\mathbf{i}} \cong \overline{B \cdot\left(s_{i_{1}} B, s_{i_{1}} s_{i_{2}} B, \ldots, w_{0} B\right)} \subset(G / B)^{l},
$$

where $B$ acts diagonally on $(G / B)^{l}$. (We give several variations of this definition below.)

This embedding of $Z_{\mathbf{i}}$ allows us to apply the tools of Standard Monomial Theory, producing a standard monomial basis for the space of sections of an effective line bundle (a graded piece of $\mathbf{C}\left[Z_{\mathbf{i}}\right]$ ). We pursue this in our papers [13], [14] with V. Lakshmibai.

In $\S 1.3$, we give another definition of the Bott-Samelson variety in terms of incidence conditions; and in $\S 1.4$, we show that the map $Z_{\mathbf{i}} \rightarrow G / B$ compactifies the matrix factorizations of Berenstein-Fomin-Zelevinsky [2], [3].

\subsection{Three constructions}

Let $W$ be the Weyl group generated by simple reflections $s_{1}, \ldots, s_{r}$, where $r$ is the rank of $G$. For $w \in W, \ell(w)$ denotes the length $l$ of a reduced (i. e. minimal) decompostion $w=s_{i_{1}} \ldots s_{i_{l}}$, and $w_{0}$ denotes the element of maximal length.

We let $B$ be a Borel subgroup, $T \subset B$ a maximal torus (Cartan subgroup). Let $P_{k} \supset B$ be the minimal parabolic associated to the simple reflection $s_{k}$, so that 
$P_{i} / B \cong \mathbf{P}^{1}$, the projective line. Also, take $\widehat{P}_{k} \supset B$ to be the maximal parabolic associated to the reflections $s_{1}, \ldots, \widehat{s_{k}}, \ldots, s_{r}$. Finally, we have the Schubert variety as a $B$-orbit closure inside the flag variety:

$$
X_{w}=\overline{B w B} \subset G / B
$$

For what follows, we fix a reduced decompostion of some $w \in W$,

$$
w=s_{i_{1}} \ldots s_{i_{l}},
$$

and we denote $\mathbf{i}=\left(i_{1}, \ldots, i_{l}\right)$.

Now let $P \supset B$ be any parabolic subgroup of $G$, and $X$ any space with $B$ action. Then the induced $P$-space is the quotient

$$
P \stackrel{B}{\times} X \stackrel{\text { def }}{=}(P \times X) / B
$$

where the quotient is by the free action of $B$ on $P \times X$ given by $(p, x) \cdot b=\left(p b, b^{-1} x\right)$. (Thus $(p b, x)=(p, b x)$ in the quotient.) The key property of this construction is that

$$
\begin{aligned}
X \rightarrow & P \\
& \stackrel{B}{\downarrow} X \\
& P / B
\end{aligned}
$$

is a fiber bundle with fiber $X$ and base $P / B$. We can iterate this construction for a sequence of parabolics $P, P^{\prime}, \ldots$,

$$
P \stackrel{B}{\times} P^{\prime} \stackrel{B}{\times} \cdots \stackrel{\text { def }}{=} P \stackrel{B}{\times}\left(P^{\prime} \stackrel{B}{\times}(\cdots)\right) .
$$

Then the quotient Bott-Samelson variety of the reduced word $\mathbf{i}$ is

$$
Z_{\mathbf{i}}^{\text {quo def }} \stackrel{\text { def }}{=} P_{i_{1}} \stackrel{B}{\times} \cdots \stackrel{B}{\times} P_{i_{l}} / B
$$

Because of the fiber-bundle property of induction, $Z_{\mathbf{i}}^{\text {quo }}$ is clearly a smooth, irreducible variety of dimension $l$. It is a subvariety of

$$
X_{l} \stackrel{\text { def }}{=} \underbrace{G \stackrel{B}{\times} \cdots \stackrel{B}{\times} G}_{l \text { factors }} / B .
$$

$B$ acts on these spaces by multiplying the first coordinate:

$$
b \cdot\left(p_{1}, p_{2}, \ldots, p_{l}\right) \stackrel{\text { def }}{=}\left(b p_{1}, p_{2}, \ldots, p_{l}\right) .
$$


The original purpose of the Bott-Samelson variety was to desingularize the Schubert variety $X_{w}$ via the multiplication map:

$$
\begin{aligned}
Z_{\mathbf{i}}^{\text {quo }} & \rightarrow X_{w} \subset G / B \\
\left(p_{1}, \ldots, p_{l}\right) & \mapsto p_{1} p_{2} \cdots p_{l} B,
\end{aligned}
$$

a birational morphism.

Next, consider the fiber product

$$
G / B \underset{G / P}{\times} G / B \stackrel{\text { def }}{=}\left\{\left(g_{1}, g_{2}\right) \in(G / B)^{2} \mid g_{1} P=g_{2} P\right\} .
$$

We may define the fiber product Bott-Samelson variety

$$
Z_{\mathbf{i}}^{\text {fib }} \stackrel{\text { def }}{=} e B \underset{G / P_{i_{1}}}{\times} G / B \underset{G / P_{i_{2}}}{\times} \cdots \underset{G / P_{i_{l}}}{\times} G / B \subset(G / B)^{l+1} .
$$

We let $B$ act diagonally on $(G / B)^{l+1}$; that is, simultaneously on each factor:

$$
b \cdot\left(g_{0} B, g_{1} B, \ldots, g_{l} B\right) \stackrel{\text { def }}{=}\left(b g_{0} B, b g_{1} B, \ldots, b g_{l} B\right) .
$$

This action restricts to $Z_{\mathbf{i}}^{\mathrm{fib}}$. The natural map to the flag variety is the projection to the last coordinate:

$$
\begin{aligned}
Z_{\mathbf{i}}^{\text {fib }} & \rightarrow G / B \\
\left(e B, g_{1} B, \ldots, g_{l} B\right) & \mapsto g_{l} B
\end{aligned}
$$

This construction is related to the correspondences of Fulton [10], Ch. 10.3.

Finally, let us define the $B$-orbit Bott-Samelson variety as the closure (in either the Zariski or analytic topologies) of the orbit of a point $z_{\mathbf{i}}$ :

$$
Z_{\mathbf{i}}^{\text {orb }} \stackrel{\text { def }}{=} \overline{B \cdot z_{\mathbf{i}}} \subset G / \widehat{P}_{i_{1}} \times \cdots \times G / \widehat{P}_{i_{l}}
$$

where

$$
z_{\mathbf{i}}=\left(s_{i_{1}} \widehat{P}_{i_{1}}, s_{i_{1}} s_{i_{2}} \widehat{P}_{i_{2}}, \ldots, s_{i_{1}} \cdots s_{i_{l}} \widehat{P}_{i_{l}}\right)
$$

Again, $B$ acts diagonally. In this case the map to $G / B$ is more difficult to describe, but see the Examples in $\S 2.3$. 


\subsection{Isomorphism theorem}

The three types of Bott-Samelson variety are isomorphic.

Theorem 1. (i) Let

$$
\phi: \begin{array}{cl}
X_{l} & \rightarrow \\
\left(g_{1}, g_{2}, \ldots, g_{l}\right) & \mapsto\left(\bar{e}, \overline{g_{1}}, \overline{g_{1} g_{2}}, \ldots, \overline{g_{1} g_{2} \cdots g_{l}}\right),
\end{array}
$$

where $\bar{g}$ means the coset of $g$. Then $\phi$ restricts to an isomorphism of B-varieties

$$
\phi: Z_{\mathbf{i}}^{\text {quo }} \stackrel{\sim}{\rightarrow} Z_{\mathbf{i}}^{\text {fib }} .
$$

(ii) Let

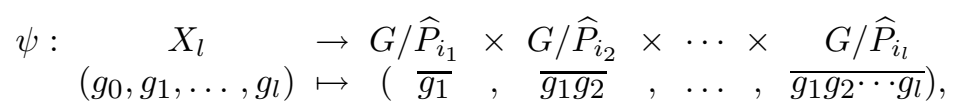

where $\bar{g}$ means the coset of $g$. Then $\psi$ restricts to an isomorphism of B-varieties

$$
\psi: Z_{\mathbf{i}}^{\text {quo }} \stackrel{\sim}{\rightarrow} Z_{\mathbf{i}}^{\text {orb }}
$$

Proof. (i) It is trivial to verify that $\phi$ is a $B$-equivariant isomorphism from $X_{l}$ to $e B \times(G / B)^{l}$ and that $\phi\left(Z_{\mathbf{i}}^{\text {quo }}\right) \subset Z_{\mathbf{i}}^{\text {fib }}$, so it suffices to show the reverse inclusion. Suppose

$$
z_{f}=\left(e B, g_{1} B, \ldots, g_{l} B\right) \in Z_{\mathbf{i}}^{\mathrm{fib}}
$$

Then

$$
z_{q}=\phi^{-1}\left(z_{f}\right)=\left(g_{1}, g_{1}^{-1} g_{2}, g_{2}^{-1} g_{3}, \ldots\right) \in X_{l}
$$

By definition, $e P_{i_{1}}=g_{1} P_{i_{1}}$, so $g_{1} \in P_{i_{1}}$. Also $g_{1} P_{i_{2}}=g_{2} P_{i_{2}}$, so $g_{1}^{-1} g_{2} \in P_{i_{2}}$, and similarly $g_{k-1}^{-1} g_{k} \in P_{i_{k}}$. Hence $z_{q} \in Z_{\mathbf{i}}^{\text {quo }}$, and $\phi\left(z_{q}\right)=z_{f}$.

(ii) First let us show that $\psi$ is injective on $Z_{\mathbf{i}}^{\text {quo }}$. Suppose $\psi\left(p_{1}, \ldots, p_{l}\right)=$ $\psi\left(q_{1}, \ldots, q_{l}\right)$ for $p_{k}, q_{k} \in P_{i_{k}}$. Then $p_{1} \widehat{P}_{i_{1}}=q_{1} \widehat{P}_{i_{1}}$, so that $p_{1}^{-1} q_{1} \in \widehat{P}_{i_{1}} \cap P_{i_{1}}=B$. Thus $q_{1}=p_{1} b_{1}$ for $b_{1} \in B$. Next, we have

$$
p_{1} p_{2} \widehat{P}_{i_{2}}=q_{1} q_{2} \widehat{P}_{i_{2}}=p_{1} b_{1} q_{2} \widehat{P}_{i_{2}}
$$

so that $p_{2}^{-1} b_{1} q_{2} \in \widehat{P}_{i_{2}} \cap P_{i_{2}}=B$, and $q_{2}=b_{1}^{-1} p_{2} b_{2}$ for $b_{2} \in B$. Continuing in this way, we find that

$$
\begin{aligned}
\left(q_{1}, q_{2}, \ldots, q_{l}\right) & =\left(p_{1} b_{1}, b_{1}^{-1} p_{2} b_{2}, \ldots, b_{l-1}^{-1} p_{l} b_{l}\right) \\
& =\left(p_{1}, p_{2}, \ldots, p_{l}\right) \in X_{l}
\end{aligned}
$$


Thus $\psi$ is injective on $Z_{\mathbf{i}}^{\text {quo }}$.

Since we are working with algebraic morphisms, we must also check that $\psi$ is injective on tangent vectors of $Z_{\mathbf{i}}^{\text {quo }}$. Now, the degeneracy locus

$$
\left\{z \in Z_{\mathbf{i}}^{\text {quo }} \mid \operatorname{Ker} d \psi_{z} \neq 0\right\}
$$

is a $B$-invariant, closed subvariety of $Z_{\mathbf{i}}^{\text {quo }}$, and by Borel's Fixed Point Theorem it must contain a $B$-fixed point. But it is easily seen that the degenerate point

$$
z_{0}=(e, \ldots, e) \in X_{l}
$$

is the only fixed point of $Z_{\mathbf{i}}^{\text {quo }}$. Thus if $d \psi$ is injective at $z_{0}$, then the degeneracy locus is empty, and $d \psi$ is injective on each tangent space. The injectivity at $z_{0}$ is easily shown by an argument completely analogous to that for global injectivity given above, but written additively in terms of Lie algebras instead of multiplicatively with Lie groups.

Thus it remains to show surjectivity: that $\psi$ takes $Z_{\mathbf{i}}^{\text {quo }}$ onto $Z_{\mathbf{i}}^{\text {orb }}$. Consider

$$
z_{\mathbf{i}}^{\text {quo }}=\left(s_{i_{1}}, \ldots, s_{i_{l}}\right) \in X_{l}
$$

a well-defined point in $Z_{\mathbf{i}}^{\text {quo }}$. Then

$$
\psi\left(z_{\mathbf{i}}^{\text {quo }}\right)=z_{\mathbf{i}}=\left(s_{i_{1}} \widehat{P}_{i_{1}}, s_{i_{1}} s_{i_{2}} \widehat{P}_{i_{2}}, \ldots\right)
$$

and $\psi$ is $B$-equivariant, so that $\psi\left(Z_{\mathbf{i}}^{\text {quo }}\right) \supset \psi\left(B \cdot z_{\mathbf{i}}^{\text {quo }}\right)=B \cdot z_{\mathbf{i}}$. However $Z_{\mathbf{i}}^{\text {quo }}$ is a projective variety, so its image under the regular map $\psi$ is closed. Hence $\psi\left(Z_{\mathbf{i}}^{\text {quo }}\right) \supset \overline{B \cdot z_{\mathbf{i}}}=Z_{\mathbf{i}}^{\text {orb }}$.

\subsection{Incidence relations}

We give another characterization of the Bott-Samelson variety as a subvariety $Z_{\mathbf{i}}^{\text {orb }} \subset G / \widehat{P}_{i_{1}} \times \cdots \times G / \widehat{P}_{i_{l}}$ in terms of certain incidence conditions, which can easily be translated into algebraic equations defining $Z_{\mathbf{i}}^{\text {orb }}$ as a variety.

Given two parabolic subgroups $P, Q \supset B$, we say the cosets $g P$ and $g^{\prime} Q$ are incident (written $g P \sim g^{\prime} Q$ ) if any of the following equivalent conditions holds:

(i) $\left(g P, g^{\prime} Q\right)$ lies in the image of the diagonal map $G /(P \cap Q) \rightarrow G / P \times G / Q$;

(ii) $g P \cap g^{\prime} Q \neq \emptyset$;

(iii) $g^{-1} g^{\prime} \in P Q$;

(iv) $g^{-1} g^{\prime} B \in X_{w}$, the Schubert variety of $G / B$ associated to the unique longest element $w$ in the set $W_{P} W_{Q} \subset W$, the product of the subgroups of $W$ corresponding to $P$ and $Q$. 
For $G=G L(n)$ and $P, Q$ maximal parabolics, the spaces $G / P, G / Q$ are Grassmannians, and our definition of incidence reduces to the inclusion relation between subspaces. (See $\S 2.3$.)

The incidence relation $\sim$ is reflexive and symmetric, but only partially transitive. One substitute for transitivity is the following property. Suppose $g_{1} Q_{1}, g_{2} Q_{2}$, $g_{3} Q_{3}$ are cosets of any parabolics with $g_{i} Q_{i} \sim g_{j} Q_{j}$ for all $i, j$. Then there exists $g_{0}$ with $g_{1} Q_{1} \sim g_{0}\left(Q_{1} \cap Q_{2}\right) \sim g_{2} Q_{2}$, and since $\left(Q_{1} \cap Q_{2}\right) Q_{3}=Q_{1} Q_{3} \cap Q_{2} Q_{3}$ (by [6], Ch. 4, Ex. 1), we conclude that $g_{0}\left(Q_{1} \cap Q_{2}\right) \sim g_{3} Q_{3}$. An immediate consequence of this property is:

Lemma 2. Consider any parabolics $Q_{1}, Q_{2}, \ldots \supset B$. Then the point $\left(g_{1} Q_{1}, g_{2} Q_{2}\right.$, ...) lies in the image of the diagonal map $G /\left(\cap_{i} Q_{i}\right) \rightarrow \prod_{i} G / Q_{i}$ if and only if $g_{i} Q_{i} \sim g_{j} Q_{j}$ for all $i, j$.

This lemma generalizes the description of $G L(n) / B$ as the variety of flags of subspaces.

The incidence relation has another transitivity property. Suppose $s, s^{\prime}, s^{\prime \prime}$ are simple reflections of $W$ such that $s^{\prime}$ is between $s$ and $s^{\prime \prime}$ in the Coxeter graph of $W$ : that is, if $s^{(1)}, s^{(2)}, \ldots, s^{(N)}$ is any sequence of simple reflections such that $s=s^{(1)}, s^{\prime \prime}=s^{(N)}$ and $s^{(j)} s^{(j+1)} \neq s^{(j+1)} s^{(j)}$ for all $j$, then $s^{\prime}=s^{(j)}$ for some $j$. Let $\widehat{P}, \widehat{P}^{\prime}, \widehat{P}^{\prime \prime}$ be the maximal parabolic subgroups of $G$ corresponding to $s, s^{\prime}, s^{\prime \prime}$. Then we may easily show that $\widehat{P} \widehat{P}^{\prime} \widehat{P}^{\prime \prime}=\widehat{P} \widehat{P}^{\prime \prime}$, so that

$$
g \widehat{P} \sim g^{\prime} \widehat{P}^{\prime} \text { and } g^{\prime} \widehat{P}^{\prime} \sim g^{\prime \prime} \widehat{P}^{\prime \prime} \Rightarrow g \widehat{P} \sim g^{\prime \prime} \widehat{P}^{\prime \prime} .
$$

From this and the previous Lemma, we obtain:

Lemma 3. Let $\widehat{P}_{1}, \ldots, \widehat{P}_{r} \supset B$ be all the maximal parabolic subgroups of $G$. Then the point $\left(g_{1} \widehat{P}_{1}, \ldots, g_{r} \widehat{P}_{r}\right)$ lies in the image of the diagonal embedding $G / B \rightarrow$ $\prod_{i=1}^{r} G / \widehat{P}_{i}$ if and only if $g_{i} \widehat{P}_{i} \sim g_{j} \widehat{P}_{j}$ for all $i, j$ with $s_{i} s_{j} \neq s_{j} s_{i}$.

To our word $\mathbf{i}=\left(i_{1}, \ldots, i_{l}\right)$ we now associate a graph $\Gamma_{\mathbf{i}}$ whose vertices are the symbols $1^{*}, 2^{*}, \ldots r^{*}$ and $1,2, \ldots, l$. (Recall that $r=\operatorname{rank} G$.) The edges of $\Gamma_{\mathbf{i}}$ are all pairs of vertices of the forms:

$$
\begin{aligned}
\left(i^{*}, k\right) & \text { with } i \neq i_{p} \text { for } 1 \leq p \leq k \text { and } s_{i} s_{i_{k}} \neq s_{i_{k}} s_{i}, \\
(j, k) & \text { with } i_{j} \neq i_{p} \text { for } j<p \leq k \text { and } s_{i_{j}} s_{i_{k}} \neq s_{i_{k}} s_{i_{j}} .
\end{aligned}
$$

The graph $\Gamma_{\mathbf{i}}$ is closely related to the wiring diagrams and chamber weights of Berenstein, Fomin, and Zelevinsky [2], [3].

Now, it follows from Theorem 1 that $Z_{\mathbf{i}}^{\text {orb }}$ is the image of $Z_{\mathbf{i}}^{\text {fib }}$ under the natural projection

$$
\begin{aligned}
(G / B)^{l+1} & \rightarrow \prod_{j=1}^{l} G / \widehat{P}_{i_{j}} \\
\left(g_{0} B, g_{1} B, \ldots, g_{l} B\right) & \mapsto\left(g_{1} \widehat{P}_{i_{1}}, \ldots, g_{l} \widehat{P}_{i_{l}}\right) .
\end{aligned}
$$


Translating this into incidence conditions using the above Lemmas, we obtain:

\section{Theorem 4.}

$$
Z_{\mathbf{i}}^{\text {orb }}=\left\{\begin{array}{l|l}
\left(g_{1} \widehat{P}_{i_{1}}, \ldots, g_{l} \widehat{P}_{i_{l}}\right) & \begin{array}{r}
e \widehat{P}_{i} \\
\sim g_{k} \widehat{P}_{i_{k}} \text { for all }\left(i^{*}, k\right) \in \Gamma_{\mathbf{i}} \\
g_{j} \widehat{P}_{i_{j}} \sim g_{k} \widehat{P}_{i_{k}} \text { for all }(j, k) \in \Gamma_{\mathbf{i}}
\end{array}
\end{array}\right\}
$$

See $\S 2.3$ below for examples in the case of $G=G L(n)$.

\subsection{Open cells and matrix factorizations}

In view of Theorem 1 , we will let $Z_{\mathbf{i}}$ denote the abstract Bott-Samelson variety defined by any of our three versions. It contains the degenerate $B$-fixed point $z_{0}$ defined by:

$$
\begin{aligned}
z_{0} & =(e, e, \ldots) \in Z_{\mathbf{i}}^{\text {quo }} \\
& =(e B, e B, \ldots) \in Z_{\mathbf{i}}^{\text {fib }} \\
& =\left(e \widehat{P}_{i_{1}}, e \widehat{P}_{i_{2}}, \ldots\right) \in Z_{\mathbf{i}}^{\text {orb }}
\end{aligned}
$$

as well as the generating $T$-fixed point whose $B$-orbit is dense in $Z_{\mathbf{i}}$ :

$$
\begin{aligned}
z_{\mathbf{i}} & =\left(s_{i_{1}}, s_{i_{2}}, s_{i_{3}}, \ldots\right) \in Z_{\mathbf{i}}^{\text {quo }} \\
& =\left(e B, s_{i_{1}} B, s_{i_{1}} s_{i_{2}} B, \ldots\right) \in Z_{\mathbf{i}}^{\text {fib }} \\
& =\left(s_{i_{1}} \widehat{P}_{i_{1}}, s_{i_{1}} s_{i_{2}} \widehat{P}_{i_{2}}, \ldots\right) \in Z_{\mathbf{i}}^{\text {orb }}
\end{aligned}
$$

Big cell. We may parametrize the dense orbit $B \cdot z_{\mathbf{i}} \subset Z_{\mathbf{i}}$ by an affine cell. Consider the normal ordering of the positive roots associated to the reduced word i. That is, let

$$
\beta_{1}=\alpha_{i_{1}}, \beta_{2}=s_{i_{1}}\left(\alpha_{i_{2}}\right), \beta_{3}=s_{i_{1}} s_{i_{2}}\left(\alpha_{i_{3}}\right), \cdots
$$

Let $U_{\beta_{k}}$ be the one-dimensional unipotent subgroup of $B$ corresponding to the positive root $\beta_{k}$. Then we have a direct product:

$$
B=U_{\beta_{1}} \cdots U_{\beta_{l}} \cdot\left(B \cap w B w^{-1}\right),
$$

so that the multiplication map

$$
\begin{aligned}
U_{\beta_{1}} \times \cdots \times U_{\beta_{l}} & \rightarrow B \cdot z_{\mathbf{i}} \\
\left(u_{1}, \ldots, u_{l}\right) & \mapsto u_{1} \cdots u_{l} \cdot z_{\mathbf{i}}
\end{aligned}
$$

is injective, and an isomorphism of varieties. The left-hand side is isomorphic to an affine space $\mathbf{C}^{l}$ (or $\mathbf{A}^{l}$ for $G$ over a general field). 
Opposite big cell. $Z_{\mathbf{i}}$ also contains an opposite big cell centered at $z_{0}$ which is not the orbit of a group. Let $U_{-\alpha_{i}}$ be the one-dimensional unipotent subgroup of $w_{0} B w_{0}$ corresponding to the negative simple root $-\alpha_{i}$. The map

$$
\begin{aligned}
\mathbf{C}^{l} \cong U_{-\alpha_{i_{1}}} \times \cdots \times U_{-\alpha_{i_{l}}} & \rightarrow Z_{\mathbf{i}}^{\text {quo }} \\
\left(u_{1}, \ldots, u_{l}\right) & \mapsto\left(u_{1}, \ldots, u_{l}\right)
\end{aligned}
$$

is an open embedding.

In the case of $G=G L(n), B=$ upper triangular matrices, we may write an element of $U_{-\alpha_{i_{k}}}$ as $u_{k}=I+t_{k} e_{k}$, where $I$ is the identity matrix, $e_{k}$ is the sub-

diagonal coordinate matrix $e_{\left(i_{k}+1, i_{k}\right)}$, and $t_{k} \in \mathbf{C}$. If we further map $Z_{\mathbf{i}}^{\text {quo }}$ to $G / B$ via the natural multiplication map, we get

$$
\begin{aligned}
& \begin{array}{ccc}
\left(t_{1}, \ldots, t_{l}\right) & \mapsto\left(I+t_{1} e_{1}\right) \cdots\left(I+t_{l} e_{l}\right) \\
\mathbf{C}^{l} & \rightarrow & N_{-}
\end{array} \\
& Z_{\mathrm{iuo}}^{\mathrm{quo}} \rightarrow \quad \cap / B \\
& \left(p_{1}, \ldots, p_{l}\right) \mapsto \quad p_{1} \cdots p_{l} B
\end{aligned}
$$

where $N_{-}$denotes the unipotent lower triangular matrices $(\bmod B)$. Thus the natural map in the bottom row compactifies the matrix factorization map in the top row, which has been studied by Berenstein, Fomin, and Zelevinsky [2]; and the corresponding statement holds in the general case of [3].

\section{Bott-Samelson varieties for $G L(n)$}

We begin again, restating our results in explicit combinatorial form for the general linear group $G=G L(n, \mathbf{C})$. We define the Bott-Samelson variety in an explicit and elementary way, which will easily show that its coordinate ring consists of generalized Schur modules. That is, a generalized Schur module bears the same relation to a Bott-Samelson variety as an ordinary (irreducible) Schur module bears to a flag variety according to the Borel-Weil Theorem. Therefore the characters, generalized Schur polyomials, can be computed by powerful Riemann-Roch type theorems just like ordinary Schur polynomials.

Our purpose in this section is to get enough combinatorial control over the BottSamelson varieties to make such theorems explicit. For a reduced decomposition $\mathbf{i}$, the Bott-Samelson variety $Z_{\mathbf{i}}$ is the space of flagged representations of a certain partially ordered set $D_{\mathbf{i}}^{+}$: that is, the variety of all embeddings of the poset $D_{\mathbf{i}}^{+}$ into the poset of subspaces of $\mathbf{C}^{n}$. (Such an embedding is flagged if a certain chain in $D_{\mathbf{i}}^{+}$maps to the standard flag $\mathbf{C}^{1} \subset \mathbf{C}^{2} \subset \cdots \mathbf{C}^{n}$.)

The posets $D_{\mathbf{i}}^{+}$can be specified by several equivalent combinatorial devices. They can be naturally embedded into the Boolean lattice of subsets of $[n]=$ $\{1,2, \ldots, n\}$. The image of such an embedding is a chamber family, associated 
to a reduced decomposition via its wiring diagram. This is easily translated into the language of generalized Young diagrams in the plane: the columns of a diagram correspond to the elements of a chamber family. It is remarkable that these different combinatorial pictures come together to describe our varieties.

In the rest of this paper, $G=G L(n)$. To make our statements more elementary, we will use $\mathbf{C}$ for our base field, but everything goes through without change over an infinite field of arbitrary characteristic or over $\mathbf{Z}$. We let $B$ be the group of invertible upper triangular matrices, $T$ the group of invertible diagonal matrices, and $\operatorname{Gr}\left(k, \mathbf{C}^{n}\right)$ the Grassmannian of $k$-dimensional subspaces of complex $n$-space. Also $W=$ permutation matrices, $\ell(w)=$ the number of inversions of a permutation $w, s_{i}=$ the transposition $(i, i+1)$, and the longest permutation is $w_{0}=n \ldots 321$. We will frequently use the notation

$$
[k]=\{1,2,3, \ldots, k\} .
$$

\subsection{Chamber families}

Define a subset family to be a collection $D=\left\{C_{1}, C_{2}, \ldots\right\}$ of subsets $C_{k} \subset[n]$. The order of the subsets is irrelevant in the family, and we do not allow subsets to be repeated.

Now suppose the list of indices $\mathbf{i}=\left(i_{1}, i_{2}, \ldots, i_{l}\right)$ encodes a reduced decomposition $w=s_{i_{1}} s_{i_{2}} \cdots s_{i_{l}}$ of a permutation into a minimal number of simple transpositions. We associate a subset family, the chamber family

$$
D_{\mathbf{i}} \stackrel{\text { def }}{=}\left\{s_{i_{1}}\left[i_{1}\right], s_{i_{1}} s_{i_{2}}\left[i_{2}\right], \ldots, w\left[i_{l}\right]\right\}
$$

Here $w[j]=\{w(1), w(2), \ldots, w(j)\}$. Further, define the full chamber family

$$
D_{\mathbf{i}}^{+} \stackrel{\text { def }}{=}\{[1],[2], \ldots,[n]\} \cup D_{\mathbf{i}}
$$

We tentatively connect these structures with geometry. Let $\mathbf{C}^{n}$ have the standard basis $e_{1}, \ldots, e_{n}$. For any subset $C=\left\{j_{1}, \ldots, j_{k}\right\} \subset[n]$, the coordinate subspace

$$
E_{C}=\operatorname{Span}_{\mathbf{C}}\left\{e_{j_{1}}, \ldots, e_{j_{k}}\right\} \in \operatorname{Gr}(k)=\operatorname{Gr}\left(k, \mathbf{C}^{n}\right)
$$

is a $T$-fixed point in a Grassmannian. A subset family corresponds to a $T$-fixed point in a product of Grassmannians

$$
z_{D}=\left(E_{C_{1}}, E_{C_{2}}, \ldots\right) \in \operatorname{Gr}(D) \stackrel{\text { def }}{=} \operatorname{Gr}\left(\left|C_{1}\right|\right) \times \operatorname{Gr}\left(\left|C_{2}\right|\right) \times \ldots
$$

We will define Bott-Samelson varieties as orbit closures of such points (see $\S 2.3$ ). 
Examples. For $n=3, G=G L(3), \mathbf{i}=121$, we have the reduced chamber family

$$
\begin{aligned}
D_{121} & =\left\{s_{1}[1], s_{1} s_{2}[2], s_{1} s_{2} s_{1}[1]\right\} \\
& =\{\{2\},\{2,3\},\{3\}\} \\
& =\{2,23,3\} .
\end{aligned}
$$

The full chamber family is $D_{121}^{+}=\{1,12,123,2,23,3\}$. The chamber family of the other reduced word $\mathbf{i}=212$ is $D_{212}=\{13,3,23\}, D_{212}^{+}=\{1,12,123,13,3,23\}$.

For $n=4$, the subset family $D=\{12,123,2,3\}$ is associated to the $T$-fixed point

$$
z_{D}=\left(E_{12}, E_{123}, E_{2}, E_{3}\right) \in \operatorname{Gr}(D)=\operatorname{Gr}(2) \times \operatorname{Gr}(3) \times \operatorname{Gr}(1) \times \operatorname{Gr}(1) .
$$

Chamber families have a rich structure. (See [18], [25].) Given a full chamber family $D_{\mathbf{i}}^{+}$, we may omit some of its elements to get a subfamily $D \subset D_{\mathbf{i}}^{+}$. The resulting chamber subfamilies can be characterized as follows.

For two sets $S, S^{\prime} \subset[n]$, we say $S$ is elementwise less than $S^{\prime}, S \stackrel{\text { dt }}{<} S^{\prime}$, if $s<s^{\prime}$ for all $s \in S, s^{\prime} \in S^{\prime}$. Now, a pair of subsets $C, C^{\prime} \subset[n]$ is strongly separated if

$$
\left(C \backslash C^{\prime}\right) \stackrel{\text { et }}{<}\left(C^{\prime} \backslash C\right) \text { or } \quad\left(C^{\prime} \backslash C\right) \stackrel{\text { et }}{<}\left(C \backslash C^{\prime}\right),
$$

where $C \backslash C^{\prime}$ denotes the complement of $C^{\prime}$ in $C$. A family of subsets is called strongly separated if each pair of subsets in it is strongly separated.

Proposition 5. (Leclerc-Zelevinsky [18]) A family D of subsets of $[n]$ is a chamber subfamily, $D \subset D_{\mathbf{i}}^{+}$for some $\mathbf{i}$, if and only if $D$ is strongly separated.

Remarks. (a) Reiner and Shimozono [25] give an equivalent description of strongly separated families. Place the subsets of the family into lexicographic order. Then $D=\left(C_{1} \stackrel{\text { lex }}{\leq} C_{2} \leq\right.$ lex $\left.\leq \cdots\right)$ is strongly separated if and only if it is "\%-avoiding": that is, if $i_{1} \in C_{j_{1}}, i_{2} \in C_{j_{2}}$ with $i_{1}>i_{2}, j_{1}<j_{2}$, then $i_{1} \in C_{j_{2}}$ or $i_{2} \in C_{j_{1}}$.

(b) If $\mathbf{i}=\left(i_{1}, \ldots, i_{l}\right)$ is an initial subword of $\mathbf{i}^{\prime}=\left(i_{1}, \ldots, i_{l}, \ldots, i_{N}\right)$, then $D_{\mathbf{i}} \subset D_{\mathbf{i}^{\prime}}$. Thus the chamber families associated to decompositions of the longest permutation $w_{0}$ are the maximal strongly separated families.

(c) In $\S 4.3$ below, we describe the "orthodontia" algorithm to determine a chamber family $D_{\mathbf{i}}^{+}$which contains to a given strongly separated family $D$. See also [25].

Examples. (a) For $n=3$, the chamber families $D_{121}^{+}=\{1,12,123,2,23,3\}$ and $D_{212}^{+}=\{1,12,123,13,3,23\}$ are the only maximal strongly separated families. The sets 13 and 2 are the only pair not strongly separated from each other.

(b) For $n=4$, the strongly separted family $D=\{24,34,4\}$ is contained in the chamber sets of the reduced words $\mathbf{i}=312132$ and $\mathbf{i}=123212$. 
1234

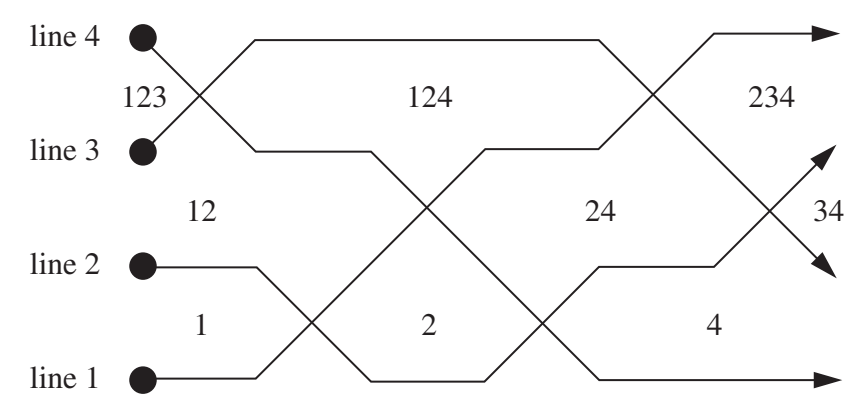

Figure 1.

\subsection{Pictures of chamber families}

Wiring diagrams. Chamber families can be represented pictorially in several ways, the most natural being due to Berenstein, Fomin, and Zelevinsky [2]. The wiring diagram or braid diagram of the reduced word $\mathbf{i}$ is best defined via an example.

Let $G=G L(4), w=w_{0}$ (the longest permutation), and $\mathbf{i}=312132$. On the right and left ends of the wiring diagram are the points $1,2,3,4$ in two columns, with 1 at the bottom and 4 at the top. Each point $i$ on the left is connected to the point $w^{-1}(i)$ on the right by a curve which is horizontal and disjoint from the other curves, except for certain X-shaped crossings. The crossings, read left to right, correspond to the entries of $\mathbf{i}$. The first entry $i_{1}=3$ corresponds to a crossing of the curve on level 3 with the one on level 4 . The curves on other levels continue horizontally. The second entry $i_{2}=1$ indicates a crossing of the curves on levels 1 and 2, the others continuing horizontally, and so on.

If we add crossings only up to the $l^{\text {th }}$ step, we obtain the wiring diagram of the truncated word $s_{i_{1}} s_{i_{2}} \cdots s_{i_{l}}$.

Now we may construct the chamber family

$$
D_{\mathbf{i}}^{+}=(1,12,123,1234,124,2,24,4,234,34)
$$

as follows. Label each of the curves of the wiring diagram by its point of origin on the left. Into each of the connected regions between the curves, write the numbers of those curves which pass below the region. Then the sets of numbers inscribed in these chambers are the members of the family $D_{\mathbf{i}}^{+}$. If we list the chambers from left to right, we recover the natural order in which these subsets appear in $D_{\mathbf{i}}^{+}$. (Warning: In BFZ's terminology, our $D_{\mathbf{i}}^{+}$would be the chamber family of the reverse word of $\mathbf{i}$, a reduced decomposition of $w^{-1}$.)

Young diagrams. Another way to picture a chamber family, or any subset family, is as follows. We may consider a subset $C=\left\{j_{1}, j_{2}, \ldots, j_{c}\right\} \subset[n]$ as a column of $c$ 
squares in the rows $j_{1}, j_{2}, \ldots$ For each subset $C_{k}$ in the chamber family, form the column associated to it, and place these columns next to each other. The result is an array of squares in the plane called a generalized Young diagram.

For our word $\mathbf{i}=312132$, we draw the (reduced) chamber family as:

$$
D_{\mathbf{i}}=\begin{array}{r}
1 \\
2 \\
3 \\
4
\end{array}
$$

where we indicate the row numbers on the left of the diagram.

\subsection{Varieties}

To any subset family $D$ we have associated a $T$-fixed point in a product of Grassmannians, $z_{D} \in \operatorname{Gr}(D)$, and we may define the configuration variety of $D$ to be the closure of the $G$-orbit of $z_{D}$ :

$$
\mathcal{F}_{D}=\overline{G \cdot z_{D}} \subset \operatorname{Gr}(D) ;
$$

and the flagged configuration variety to be the closure of its $B$-orbit:

$$
\mathcal{F}_{D}^{B}=\overline{B \cdot z_{D}} \subset \operatorname{Gr}(D) .
$$

Furthermore, if $D=D_{\mathbf{i}}$, a chamber family, then the Bott-Samelson variety is the flagged configuration variety of $D_{\mathbf{i}}$ :

$$
Z_{\mathbf{i}}=Z_{\mathbf{i}}^{\text {orb }}=\mathcal{F}_{D}^{B}
$$

(We could also use the full chamber family $D_{\mathbf{i}}^{+}$, since the extra coordinates correspond to the standard flag fixed under the $B$-action.)

Thus $\mathcal{F}_{D}, \mathcal{F}_{D}^{B}$, and $Z_{\mathbf{i}}$ can be considered as varieties of configurations of subspaces in $\mathbf{C}^{n}$, like the flag and Schubert varieties. We will give defining equations for the Bott-Samelson varieties analogous to those for Schubert varieties.

For a subset family $D$ with partial order given by inclusion, define the variety of flagged representations of $D$

$$
\mathcal{R}_{D}^{B}=\left\{\begin{array}{l|c}
\left(V_{C}\right)_{C \in D} \in \operatorname{Gr}(D) & \begin{array}{c}
\forall C, C^{\prime} \in D, C \subset C^{\prime} \Rightarrow V_{C} \subset V_{C^{\prime}} \\
\text { and } \forall[i] \in D, \quad V_{[i]}=\mathbf{C}^{i}
\end{array}
\end{array}\right\} .
$$

("Flagged" refers to the condition that a space $V_{[i]}$ corresponding to an initial interval $[i] \in D$ is fixed to be an element of the standard flag $\mathbf{C}^{1} \subset \mathbf{C}^{2} \subset \cdots$.) Let $B$ act diagonally on $\mathcal{R}_{D}^{B}$.

The following proposition is a special case of Prop. 4 of $\S 1.3$.

Proposition 6. For every reduced word $\mathbf{i}$, we have $Z_{\mathbf{i}} \cong \mathcal{R}_{D^{+}}^{B}$. 


\subsection{Examples of varieties}

Example. For $n=4, \mathbf{i}=312132$, we may use the picture in the above example to write the Bott-Samelson variety $Z_{\mathbf{i}}=\mathcal{R}_{D^{+}}^{B}$ as the set of all 6-tuples of subspaces of $\mathbf{C}^{4},\left(V_{124}, V_{2}, V_{24}, V_{4}, V_{234}, V_{34}\right)$ with $\operatorname{dim}\left(V_{C}\right)=|C|$ and satisfying the following inclusions:

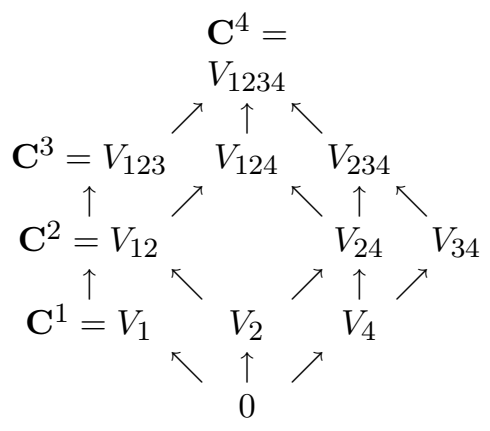

where the arrows indicate inclusion of subspaces. The natural map onto the flag variety projects $\left(V_{124}, \ldots, V_{34}\right)$ to the flag at the right edge of the picture: $(0 \subset$ $\left.V_{4} \subset V_{34} \subset V_{234} \subset \mathbf{C}^{4}\right)$.

Example. Desingularizing a Schubert variety. Let $n=7$, and consider the family $D$ comprising the single subset $C=12457$. Its configuration variety is the Grassmannian $\mathcal{F}_{D}=\operatorname{Gr}\left(5, \mathbf{C}^{7}\right)$, and its flagged configuration variety is a Schubert variety $X_{\lambda}$ in this Grassmannian:

$$
\mathcal{F}_{D}^{B}=X_{\lambda}=\left\{V \in \operatorname{Gr}\left(5, \mathbf{C}^{7}\right) \mid \mathbf{C}^{2} \subset V, \operatorname{dim}\left(\mathbf{C}^{5} \cap V\right) \geq 4\right\} .
$$

Here the indexing partition $\lambda=(0,0,1,1,2)$ is obtained from the subset $C=12457$ by subtracting $1,2, \ldots$ from its elements: $0=1-1,0=2-2,1=4-3$, $1=5-4,2=7-5$.) Now, we know by Proposition 5 that any strongly separated family is part of some chamber family $D_{\mathbf{i}}$. In fact, we may take $\mathbf{i}$ so that the projection map $Z_{\mathbf{i}}=\mathcal{F}_{D}^{B} \rightarrow \mathcal{F}_{D}^{B}$ is birational. The orthodontia algorithm of $\S 4.3$ below produces such an $\mathbf{i}$.

By orthodontia, we find that our variety is desingularized by the reduced word $\mathbf{i}=3465$, for which $D_{\mathbf{i}}=\{124,1245,123457,12457\}$ and

$$
Z_{\mathbf{i}}=\left\{\begin{array}{c}
\left(V_{124}, V_{1245}, V_{123457}, V_{12457}\right) \in \mathrm{Gr}(3) \times \mathrm{Gr}(4) \times \mathrm{Gr}(6) \times \mathrm{Gr}(5) \\
\text { such that } \quad \mathbf{C}^{2} \subset V_{124} \subset \mathbf{C}^{4} \subset V_{123457}, \quad V_{1245} \subset \mathbf{C}^{5}, \\
V_{124} \subset V_{1245} \subset V_{12457} \subset V_{123457}
\end{array}\right\} .
$$

The desingularization map is the projection

$$
\pi: \begin{array}{cc}
Z_{\mathbf{i}} & \rightarrow \mathcal{F}_{D}^{B}=X_{\lambda} \\
\left(V_{124}, V_{1245}, V_{123457}, V_{12457}\right) & \mapsto V_{12457} .
\end{array}
$$


In our paper [21] and Zelevinsky's work [26], there are given other desingularizations of Schubert varieties, all of them expressible as flagged configuration varieties.

Conjecture 7. For any subset family $D$, a configuration $\left(V_{C}\right)_{C \in D} \in \operatorname{Gr}(D)$ lies in $\mathcal{F}_{D}$ exactly if, for every subfamily $D^{\prime} \subset D$,

$$
\begin{aligned}
& \operatorname{dim}\left(\bigcap_{C \in D^{\prime}} V_{C}\right) \geq\left|\cap_{C \in D^{\prime}} C\right| \\
& \operatorname{dim}\left(\sum_{C \in D^{\prime}} V_{C}\right) \leq\left|\cup_{C \in D^{\prime}} C\right|
\end{aligned}
$$

Remarks. (a) If $D=D_{\mathbf{i}}$ is a chamber family, the conjecture reduces to the previous theorem.

(b) The conjecture is known if $D$ satisfies the "northwest condition" (see [21]): that is, the elements of $D$ can be arranged in an order $C_{1}, C_{2}, \ldots$ such that if $i_{1} \in C_{j_{1}}, i_{2} \in C_{j_{2}}$, then $\min \left(i_{1}, i_{2}\right) \in C_{\min \left(j_{1}, j_{2}\right)}$. In fact, it suffices in this case to consider only the intersection conditions of the conjecture.

(c) Note that a configuration $\left(V_{1}, \ldots, V_{l}\right) \in \operatorname{Gr}(D)$ lies in the flagged configuration variety $\mathcal{F}_{D}^{B}$ if and only if $\left(\mathbf{C}^{1}, \ldots, \mathbf{C}^{n}, V_{1}, \ldots, V_{l}\right)$ lies in the unflagged variety $\mathcal{F}_{D^{+}}$ of the augmented diagram $D^{+} \stackrel{\text { def }}{=}\{[1],[2], \ldots[n]\} \cup D$. Hence the conjecture gives conditions defining flagged configuration varieties as well as unflagged.

(d) It would be interesting to know whether the determinantal equations implied by the conditions of the conjecture (and the previous theorem) define $\mathcal{F}_{D} \subset \operatorname{Gr}(D)$ scheme-theoretically.

\section{Schur and Weyl modules}

The most familiar construction of Schur modules is in terms of Young symmetrizers acting on a large tensor power of $\mathbf{C}^{n}$. This construction is limited to characteristic zero, however, so we use an alternative construction in the spirit of DeRuyts [10], Desarmenien-Kung-Rota [9], and Carter-Lusztig. This construction is universally valid and is more natural geometrically. (We sketch the connection with the symmetrizer picture at the end of §3.1.) Using the same arguments as in [21], our Borel-Weil Theorem is immediate, and we work out a version of Demazure's character formula to get a new expression for generalized Schur polynomials.

\subsection{Definitions}

We have associated to any subset family $D=\left\{C_{1}, \ldots, C_{k}\right\}$ a configuration variety $\mathcal{F}_{D}$ with $G$-action, and a flagged configuration variety $\mathcal{F}_{D}^{B}$ with $B$-action. Now, assign an integer multiplicity $\mathbf{m}(C) \geq 0$ to each subset $C \in D$. For each pair 
$(D, \mathbf{m})$, we define a $G$-module and a $B$-module, which will turn out to sections of a line bundle on $\mathcal{F}_{D}$ and $\mathcal{F}_{D}^{B}$. We construct these "Weyl modules" $M_{D, \mathbf{m}}$ inside the coordinate ring of $n \times n$ matrices, and their flagged versions $M_{D, \mathbf{m}}^{B}$ inside the coordinate ring of upper-triangular matrices.

Let $\mathbf{C}\left[x_{i j}\right]$ (resp. $\mathbf{C}\left[x_{i j}\right]_{i \leq j}$ ) denote the polynomial functions in the variables $x_{i j}$ with $i, j \in[n]\left(\right.$ resp. $x_{i j}$ with $\left.1 \leq i \leq j \leq n\right)$. For $R, C \subset[n]$ with $|R|=|C|$, let

$$
\Delta_{C}^{R}=\operatorname{det}\left(x_{i j}\right)_{(i \in R, j \in C)} \in \mathbf{C}\left[x_{i j}\right]
$$

be the minor of the matrix $x=\left(x_{i j}\right)$ on the rows $R$ and the columns $C$. Further, let

$$
\widetilde{\Delta}_{C}^{R}=\left.\Delta_{C}^{R}\right|_{x_{i j}=0, \forall i>j} \in \mathbf{C}\left[x_{i j}\right]_{i \leq j}
$$

be the same minor evaluated on an upper triangular matrix of variables.

Now, for a subset family $D=\left\{C_{1}, \ldots, C_{l}\right\}, \mathbf{m}=\left(m_{1}, \ldots, m_{l}\right)$, define the Weyl module

$$
M_{D, \mathbf{m}}=\operatorname{Span}_{\mathbf{C}}\left\{\begin{array}{l|l}
\Delta_{C_{1}}^{R_{11}} \cdots \Delta_{C_{1}}^{R_{1 m_{1}}} \Delta_{C_{2}}^{R_{21}} \ldots \Delta_{C_{l}}^{R_{l m_{l}}} \mid \begin{array}{ll}
\forall k, m & R_{k m} \subset[n] \\
\text { and }\left|R_{k m}\right|=\left|C_{k}\right|
\end{array}
\end{array}\right\} .
$$

That is, a spanning vector is a product of minors with column indices equal to the elements of $D$ and row indices taken arbitrarily.

For two sets $R=\left\{i_{1}<\cdots<i_{c}\right\}, C=\left\{j_{1}<\cdots<j_{c}\right\}$ we say $R \stackrel{\text { comp }}{\leq} C$ (componentwise inequality) if $i_{1} \leq j_{1}, i_{2} \leq j_{2}, \ldots$. Define the flagged Weyl module

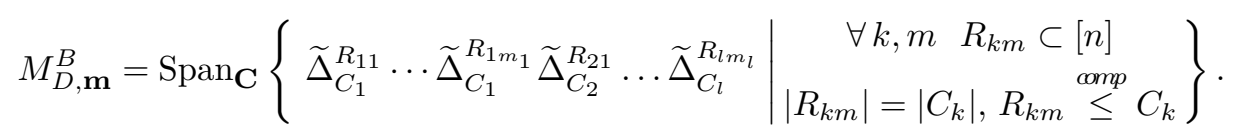

(In fact, the condition $R_{k m} \stackrel{\text { comp }}{\leq} C_{k}$ is superfluous, since $\widetilde{\Delta}_{C}^{R}=0$ unless $R_{k m} \stackrel{\text { comp }}{\leq}$ $C_{k}$.)

For $f(x) \in \mathbf{C}\left[x_{i j}\right]$, a matrix $g \in G$ acts by left translation, $(g \cdot f)(x)=f\left(g^{-1} x\right)$. It is easily seen that this restricts to a $G$-action on $M_{D, \mathbf{m}}$ and similarly we get a $B$-action on $M_{D, \mathbf{m}}^{B}$.

We clearly have the diagram of $B$-modules:

$$
\begin{array}{ccc}
M_{D, \mathbf{m}} & \subset & \mathbf{C}\left[x_{i j}\right] \\
\downarrow & & \downarrow \\
M_{D, \mathbf{m}}^{B} & \subset & \mathbf{C}\left[x_{i j}\right]_{i \leq j}
\end{array}
$$

where the vertical maps $\left(x_{i j} \mapsto 0\right.$ for $\left.i>j\right)$ are surjective. That is, $M_{D, \mathbf{m}}^{B}$ is a quotient of $M_{D, \mathbf{m}}$. 
The Schur modules are defined to be the duals

$$
S_{D, \mathbf{m}} \stackrel{\text { def }}{=}\left(M_{D, \mathbf{m}}\right)^{*} \quad S_{D, \mathbf{m}}^{B} \stackrel{\text { def }}{=}\left(M_{D, \mathbf{m}}^{B}\right)^{*} .
$$

We will deal mostly with the Weyl modules, but everything we say will of course have a dual version applying to Schur modules.

Example. Let $n=4, D=\{234,34,4\}, \mathbf{m}=(2,0,3)$. (That is, $m(234)=2$, $m(34)=0, m(4)=3$.) We picture this as a generalized Young diagram by writing each column repeatedly, according to its multiplicity. Zero multiplicity means we omit the column. Thus

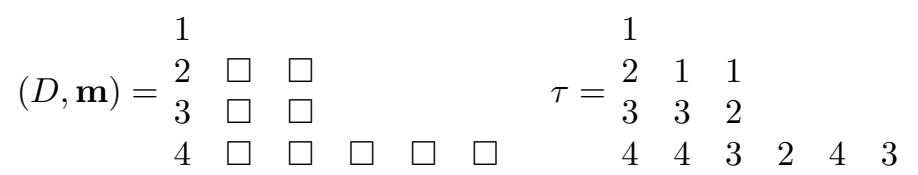

The spanning vectors for $M_{D, \mathbf{m}}$ correspond to all column-strict fillings of this diagram by indices in $[n]$. For example, the filling $\tau$ above corresponds to

$$
\begin{aligned}
& \Delta_{234}^{134} \Delta_{234}^{123} \Delta_{4}^{2} \Delta_{4}^{4} \Delta_{4}^{3} \\
& =\left|\begin{array}{lll}
x_{12} & x_{13} & x_{14} \\
x_{32} & x_{33} & x_{34} \\
x_{42} & x_{43} & x_{44}
\end{array}\right| \cdot\left|\begin{array}{lll}
x_{12} & x_{13} & x_{14} \\
x_{22} & x_{23} & x_{24} \\
x_{32} & x_{33} & x_{34}
\end{array}\right| \cdot x_{24} \cdot x_{44} \cdot x_{34} \\
& =\left(\begin{array}{llll|llllll}
1 & 1 & & & & 2 & 2 & & & \\
3 & 2 & & & & 3 & & & \\
4 & 3 & 2 & 4 & 3 & 4 & 4 & 4 & 4
\end{array}\right)
\end{aligned}
$$

The last expression is in the letter-place notation of Rota et al [9].

A basis may be extracted from this spanning set by considering only the rowdecreasing fillings (a normalization of the semi-standard tableaux), and in fact the Weyl module is the dual of the classical Schur module $S_{\lambda}$ associated to the shape $D$ considered as the Young diagram $\lambda=(0,2,2,5)$.

The spanning elements of the flagged Weyl module $M_{D, \mathbf{m}}^{B}$ correspond to the "flagged" fillings of the diagram: those for which the number $i$ does not appear above the $i^{\text {th }}$ level. For the diagram above, all the column-strict fillings are flagged, and $M_{D, \mathbf{m}} \cong M_{D, \mathbf{m}}^{B}$.

However, for

$$
\left(D^{\prime}, \mathbf{m}\right)=\begin{aligned}
& 1 \\
& 2 \\
& 3 \\
& 4
\end{aligned}
$$




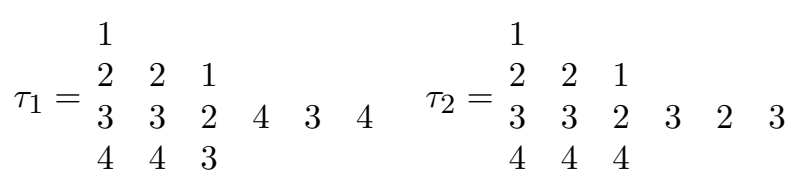

the filling $\tau_{1}$ is not flagged, since 4 appears on the 3 rd level, but $\tau_{2}$ is flagged, and corresponds to the spanning element

$$
\widetilde{\Delta}_{234}^{234} \widetilde{\Delta}_{234}^{124} \widetilde{\Delta}_{3}^{3} \widetilde{\Delta}_{3}^{2} \widetilde{\Delta}_{3}^{3}=\left|\begin{array}{ccc}
x_{22} & x_{23} & x_{24} \\
0 & x_{33} & x_{34} \\
0 & 0 & x_{44}
\end{array}\right| \cdot\left|\begin{array}{ccc}
x_{12} & x_{13} & x_{14} \\
x_{22} & x_{23} & x_{24} \\
0 & 0 & x_{44}
\end{array}\right| \cdot x_{33} \cdot x_{23} \cdot x_{33} .
$$

We have $M_{D, \mathbf{m}} \cong M_{D^{\prime}, \mathbf{m}} \cong M_{D, \mathbf{m}}^{B} \cong S_{(0,2,2,5)}^{*}$, the dual of a classical (irreducible) Schur module for $G L(4)$, and $M_{D^{\prime}, \mathbf{m}}^{B} \cong S_{(0,2,5,2)}^{*}$, the dual of the Demazure module with lowest weight $(0,2,5,2)$ and highest weight $(5,2,2,0)$. Cf. $[23]$.

Remarks. (a) In [13], [14] and $\S 4.4$ below, we make a general definition of "standard tableaux" giving bases of the Weyl modules for strongly separated families. (b) We briefly indicate the equivalence between our definition of the Weyl modules and the tensor product definition given in [1], [23], [21].

Let $Y=Y_{D, \mathbf{m}} \subset \mathbf{N} \times \mathbf{N}$ be the generalized Young diagram of squares in the plane associated to $(D, \mathbf{m})$ as in the above examples, and let $U=\left(\mathbf{C}^{n}\right)^{*}$. One defines $M_{Y}^{\text {tensor }}=U^{\otimes Y} \gamma_{Y}$, where $\gamma_{Y}$ is a generalized Young symmetrizer. The spanning vectors $\Delta_{\tau}$ of $M_{D, \mathbf{m}}$ correspond to the fillings $\tau: Y \rightarrow[n]$. Then the map

$$
\begin{aligned}
M_{D, \mathbf{m}} & \rightarrow M_{Y}^{\text {tensor }} \\
\Delta_{\tau} & \mapsto\left(\bigotimes_{(i, j) \in Y} e_{\tau(i, j)}^{*}\right) \gamma_{Y}
\end{aligned}
$$

is a well-defined isomorphism of $G$-modules, and similarly for the flagged versions. This is easily seen from the definitions, and also follows from the Borel-Weil theorems proved below and in [21].

\subsection{Borel-Weil theory}

A configuration variety $\mathcal{F}_{D} \subset \operatorname{Gr}(D)$ has a natural family of line bundles defined by restricting the determinant or Plucker bundles on the factors of $\operatorname{Gr}(D)$. For $D=\left(C_{1}, C_{2}, \ldots\right)$, and multiplicities $\mathbf{m}=\left(m_{1}, m_{2}, \ldots\right)$, we define

$$
\begin{array}{ccc}
\mathcal{L}_{\mathbf{m}} & \subset & \mathcal{O}\left(m_{1}, m_{2}, \ldots\right) \\
\downarrow & \downarrow & \downarrow \\
\mathcal{F}_{D} & \subset & \operatorname{Gr}(D)=\operatorname{Gr}\left(\left|C_{1}\right|\right) \times \operatorname{Gr}\left(\left|C_{2}\right|\right) \\
\times \cdots & &
\end{array}
$$


We denote by the same symbol $\mathcal{L}_{\mathbf{m}}$ this line bundle restricted to $\mathcal{F}_{D}^{B}$. Note that in the case of a Bott-Samelson variety $\mathcal{F}_{D}=Z_{\mathbf{i}}$, this is the well-known line bundle

$$
\begin{gathered}
\mathcal{L}_{\mathbf{m}} \cong \frac{P_{i_{1}} \times \cdots \times P_{i_{l}} \times \mathbf{C}}{B^{l}} \\
\left(p_{1}, \ldots, p_{l}, v\right) \cdot\left(b_{1}, \ldots, b_{l}\right) \stackrel{\text { def }}{=}\left(p_{1} b_{1}, \ldots, b_{l-1}^{-1} p_{l} b_{l}, \varpi_{i_{1}}\left(b_{1}^{-1}\right)^{m_{1}} \cdots \varpi_{i_{l}}\left(b_{l}^{-1}\right)^{m_{l}} v\right),
\end{gathered}
$$

$\varpi_{i}$ denoting the fundamental weight $\varpi_{i}\left(\operatorname{diag}\left(x_{1}, \ldots, x_{n}\right)\right)=x_{1} x_{2} \cdots x_{i}$.

Note that if $m_{k} \geq 0$ for all $k$ (resp. $m_{k}>0$ for all $k$ ) then $\mathcal{L}_{\mathbf{m}}$ is effective (resp. very ample). However, $\mathcal{L}_{\mathbf{m}}$ may be effective even if some $m_{k}<0$.

Proposition 8. Let $(D, \mathbf{m})$ be a strongly separated subset family with multiplicity. Then we have

(i) $M_{D, \mathbf{m}} \cong H^{0}\left(\mathcal{F}_{D}, \mathcal{L}_{\mathbf{m}}\right)$ and $H^{i}\left(\mathcal{F}_{D}, \mathcal{L}_{\mathbf{m}}\right)=0$ for $i>0$.

(ii) $M_{D, \mathbf{m}}^{B} \cong H^{0}\left(\mathcal{F}_{D}^{B}, \mathcal{L}_{\mathbf{m}}\right)$ and $H^{i}\left(\mathcal{F}_{D}^{B}, \mathcal{L}_{\mathbf{m}}\right)=0$ for $i>0$.

(iii) $\mathcal{F}_{D}$ and $\mathcal{F}_{D}^{B}$ are normal varieties, projectively normal with respect to $\mathcal{L}_{\mathbf{m}}$, and have rational singularities.

Proof. First, recall that we can identify the sections of a bundle over a single Grassmannian, $\mathcal{O}(1) \rightarrow \operatorname{Gr}(i)$, with linear combinations of $i \times i$ minors $\Delta^{R}(x)$ in the homogeneous Stiefel coordinates

$$
x=\left(\begin{array}{ccc}
x_{11} & \cdots & x_{1 i} \\
\vdots & \ddots & \vdots \\
x_{n 1} & \cdots & x_{n i}
\end{array}\right) \in \operatorname{Gr}(i)
$$

where $R$ denotes any set of row indices $R \subset[n],|R|=i$. Thus, a typical spanning element of $H^{0}(\operatorname{Gr}(D), \mathcal{O}(\mathbf{m}))$ is the section

$$
\Delta^{R_{11}}\left(x^{(1)}\right) \cdots \Delta^{R_{11}}\left(x^{(1)}\right) \Delta^{R_{21}}\left(x^{(2)}\right) \cdots \Delta^{R_{l m_{l}}\left(x^{(l)}\right),}
$$

where $x^{(k)}$ represents the homogeneous coordinates on each factor $\operatorname{Gr}\left(\left|C_{k}\right|\right)$ of $\operatorname{Gr}(D)$, and $R_{k m}$ are arbitrary subsets with $\left|R_{k m}\right|=i_{k}$.

Now, restrict the above section to $\mathcal{F}_{D} \subset \operatorname{Gr}(D)$ and then further to the dense $G$-orbit $G \cdot z_{D} \subset \mathcal{F}_{D}$. Parametrizing the orbit by $g \mapsto g \cdot z_{D}$, we pull back the resulting sections of $H^{0}\left(\mathcal{F}_{D}, \mathcal{L}_{\mathbf{m}}\right)$ to certain functions on $G \subset \operatorname{Mat}_{n \times n}(\mathbf{C})$, which are precisely the products of minors defining the spanning set of $M_{D, \mathbf{m}}$. This shows that

$$
M_{D, \mathbf{m}} \cong \operatorname{Im}\left[H^{0}(\operatorname{Gr}(D), \mathcal{O}(\mathbf{m})) \stackrel{\text { rest }}{\rightarrow} H^{0}\left(\mathcal{F}_{D}, \mathcal{L}_{\mathbf{m}}\right)\right] .
$$

Similarly for $B$-orbits, we have

$$
M_{D, \mathbf{m}}^{B} \cong \operatorname{Im}\left[H^{0}(\operatorname{Gr}(D), \mathcal{O}(\mathbf{m})) \stackrel{\text { rest }}{\rightarrow} H^{0}\left(\mathcal{F}_{D}^{B}, \mathcal{L}_{\mathbf{m}}\right)\right] .
$$


Given this description of $M_{D, \mathbf{m}}$, our Proposition becomes a restatement of the vanishing results in [21], Props. 25 and 28 (due to W. van der Kallen and S.P. Inamdar, applying the work of O. Mathieu [22], P. Polo, et.al.). The conditions $(\alpha)$ and $(\beta)$ of these propositions apply to $\mathcal{F}_{D}$ because $D$ is contained in a chamber family $D_{i}^{+}$(Prop. 5 above). Furthermore, the proof of [21], Props. 25 and 28 go through identically with $\mathcal{F}_{D}^{B}$ in place of $\mathcal{F}_{D}$, merely replacing $\mathcal{F}_{w_{0} ; u_{1}, \ldots, u_{r}}$ by $\mathcal{F}_{e ; u_{1}, \ldots, u_{r}}$.

We recall another result from [21]: For the unflagged case, the following proposition is a restatement of [21], Prop. 28(a). Again, the proof given there goes through almost identically for the flagged case.

Proposition 9. Suppose $(D, \mathbf{m}),(\widetilde{D}, \widetilde{\mathbf{m}})$ are strongly separated subset families with $D \subset \widetilde{D}, \widetilde{\mathbf{m}}(C)=\mathbf{m}(C)$ for $C \in D, \mathbf{m}(C)=0$ otherwise. Then the natural projection $\pi: \operatorname{Gr}(\widetilde{D}) \rightarrow \operatorname{Gr}(D)$ restricts to a surjection $\pi: \mathcal{F}_{\widetilde{D}} \rightarrow \mathcal{F}_{D}$, and induces an isomorphism

$$
\pi^{*}: H^{0}\left(\mathcal{F}_{D}, \mathcal{L}_{\mathbf{m}}\right) \stackrel{\sim}{\rightarrow} H^{0}\left(\mathcal{F}_{\widetilde{D}}, \mathcal{L}_{\widetilde{\mathbf{m}}}\right)
$$

and similarly for the flagged case.

Remarks. (a) Note that the proposition holds even if $\operatorname{dim} \mathcal{F}_{\widetilde{D}}>\operatorname{dim} \mathcal{F}_{D}$.

(b) We will use the proposition in the case where $D$ is a strongly separated family which is part of the chamber family $\widetilde{D}=D_{\mathbf{i}}$. The above propositions give:

$$
M_{D, \mathbf{m}} \cong H^{0}\left(\mathcal{F}_{D}, \mathcal{L}_{\mathbf{m}}\right) \cong H^{0}\left(\mathcal{F}_{D}, \mathcal{L}_{\widetilde{\mathbf{m}}}\right)=H^{0}\left(Z_{\mathbf{i}}, \mathcal{L}_{\widetilde{\mathbf{m}}}\right) .
$$

In the next section, we apply the Demazure formula for Bott-Samelson varieties to compute the character of $M_{D, \mathbf{m}}$.

(c) We may conjecture that all the results of this section hold not only in the strongly separated case, but for all subset families and configuration varieties.

\subsection{Demazure character formula}

We now examine how the iterative structure of Bott-Samelson varieties helps to understand the associated Weyl modules.

Define Demazure's isobaric divided difference operator $\Lambda_{i}: \mathbf{C}\left[x_{1}, \ldots, x_{n}\right] \rightarrow$ $\mathbf{C}\left[x_{1}, \ldots, x_{n}\right]$,

$$
\Lambda_{i} f=\frac{x_{i} f-x_{i+1} s_{i} f}{x_{i}-x_{i+1}} .
$$

For example for $f\left(x_{1}, x_{2}, x_{3}\right)=x_{1}^{2} x_{2}^{2} x_{3}$,

$$
\begin{aligned}
\Lambda_{2} f\left(x_{1}, x_{2}, x_{3}\right) & =\frac{x_{2}\left(x_{1}^{2} x_{2}^{2} x_{3}\right)-x_{3}\left(x_{1}^{2} x_{3}^{2} x_{2}\right)}{x_{2}-x_{3}} \\
& =x_{1}^{2} x_{2} x_{3}\left(x_{2}+x_{3}\right) .
\end{aligned}
$$


For any permutation with a reduced decompostion $w=s_{i_{1}} \ldots s_{i_{l}}$, define

$$
\Lambda_{w} \stackrel{\text { def }}{=} \Lambda_{i_{1}} \cdots \Lambda_{i_{l}},
$$

which is known to be independent of the reduced decomposition chosen.

By the dual character of a $G$ - or $B$-module $M$, we mean

$$
\operatorname{char}^{*} M=\operatorname{tr}\left(\operatorname{diag}\left(x_{1}, \ldots, x_{n}\right) \mid M^{*}\right) \in \mathbf{C}\left[x_{1}^{ \pm 1}, \ldots, x_{n}^{ \pm 1}\right] .
$$

(The dual character of a Weyl module is the ordinary character of the corresponding Schur module, a polynomial in $x_{1}, x_{2}, \ldots$ ) Let $\varpi_{i}$ denote the $i$ th fundamental weight, the multiplicative character of $B$ defined by $\varpi_{i}\left(\operatorname{diag}\left(x_{1}, \ldots, x_{n}\right)\right)=$ $x_{1} x_{2} \cdots x_{i}$.

Proposition 10. Suppose $(D, \mathbf{m})$ is strongly separated, and

$$
D \subset D_{\mathbf{i}}^{+}=\left\{[1], \ldots,[n], C_{1}, \ldots, C_{l}\right\},
$$

for some reduced word $\mathbf{i}=\left(i_{1}, \ldots, i_{l}\right)$. Define $\widetilde{\mathbf{m}}=\left(k_{1}, \ldots, k_{n}, m_{1}, \ldots, m_{l}\right)$ by $\widetilde{\mathbf{m}}(C)=\mathbf{m}(C)$ for $C \in D, \widetilde{\mathbf{m}}(C)=0$ otherwise. Then

$$
\operatorname{char}^{*} M_{D, \mathbf{m}}^{B}=\varpi_{1}^{k_{1}} \ldots \varpi_{n}^{k_{n}} \Lambda_{i_{1}}\left(\varpi_{i_{1}}^{m_{1}} \ldots\left(\Lambda_{i_{l}} \varpi_{i_{l}}^{m_{l}}\right) \ldots\right) .
$$

Furthermore,

$$
\operatorname{char}^{*} M_{D, \mathbf{m}}=\Lambda_{w_{0}} \operatorname{char}^{*} M_{D, \mathbf{m}}^{B},
$$

where $w_{0}$ denotes the longest permutation.

Remark. We explain in $\S 4.4$ below (and in [13], [14]) how one can recursively generate a set of standard tableaux for $M_{D}^{B}$ by "quantizing" this character formula.

We devote the rest of this section to proving the Proposition.

For a subset $C=\left\{j_{1}, j_{2}, \ldots\right\} \subset[n]$, and a permutation $w$, let $w C=\left\{w\left(j_{1}\right)\right.$, $\left.w\left(j_{2}\right), \ldots\right\}$, and for a subset family $D=\left\{C_{1}, C_{2}, \ldots\right\}$, let $w D=\left\{w C_{1}, w C_{2}, \ldots\right\}$. Now, for $i \in[n-1]$, let

$$
\Lambda_{i} D \stackrel{\text { def }}{=}\left\{s_{i}[i]\right\} \cup s_{i} D,
$$

where $s_{i}[i]=\{1,2, \ldots, i-1, i+1\}$. We say that $D$ is $i$-free for $i \in[n]$ if for every $C \in D$, we have $C \cap\{i, i+1\} \neq\{i+1\}$.

Lemma 11. Suppose $(D, \mathbf{m})$ is strongly separated and $i$-free. Then:

(i) $\mathcal{F}_{\Lambda_{i} D}^{B} \cong P_{i} \times{ }^{B} \mathcal{F}_{D}^{B}$.

(ii) $\mathcal{F}_{s_{i} D}^{B} \cong P_{i} \cdot \mathcal{F}_{D}^{B} \subset \operatorname{Gr}(D)$.

(iii) The projection $\mathcal{F}_{\Lambda_{i} D}^{B} \rightarrow \mathcal{F}_{s_{i} D}^{B}$ is regular, surjective, and birational. 
(iv) Let $\widetilde{\mathbf{m}}$ be the multiplicity on $\Lambda_{i} D$ defined by $\widetilde{\mathbf{m}}\left(s_{i} C\right)=\mathbf{m}(C)$ for $C \in D$, $\widetilde{\mathbf{m}}\left(s_{i}[i]\right)=m_{0}$. The bundle $\mathcal{L}_{\widetilde{\mathbf{m}}} \rightarrow \mathcal{F}_{\Lambda_{i} D}^{B}$ is isomorphic to

$$
\mathcal{L}_{\widetilde{\mathbf{m}}} \cong P_{i} \stackrel{B}{\times}\left(\left(\varpi_{i}^{m_{0}}\right)^{*} \otimes \mathcal{L}_{\mathbf{m}}\right),
$$

where $\left(\varpi_{i}^{m_{0}}\right)^{*} \otimes \mathcal{L}_{\mathbf{m}}$ indicates the bundle $\mathcal{L}_{\mathbf{m}} \rightarrow \mathcal{F}_{D}^{B}$ with its B-action twisted by the multiplicative character $\left(\varpi_{i}^{m_{0}}\right)^{*}=\varpi_{i}^{-m_{0}}$.

Proof. (i) Since $D$ is $i$-free, we have $U_{i} z_{D}=z_{D}$, where $U_{i}$ is the one-dimensional unipotent subgroup corresponding to the simple root $\alpha_{i}$. We may factor $B$ into a direct product of subgroups, $B=U_{i} B^{\prime}=B^{\prime} U_{i}$. Then

$$
\mathcal{F}_{D}^{B}=\overline{B \cdot z_{D}}=\overline{B^{\prime} \cdot z_{D}}
$$

Hence the $T$-fixed point $\left(s_{i}, z_{D}\right) \in P_{i} \stackrel{B}{\times} \mathcal{F}_{D}^{B}$ has a dense $B$-orbit:

$$
\begin{aligned}
\overline{B \cdot\left(s_{i}, z_{D}\right)} & =\overline{\left(U_{i} B^{\prime} s_{i}, z_{D}\right)} \\
& \left.=\overline{\left(U_{i} s_{i}\right.}, \overline{B^{\prime} \cdot z_{D}}\right) \\
& =P_{i} \times \mathcal{F}_{D}^{B} .
\end{aligned}
$$

Clearly, the injective map

$$
\begin{aligned}
\psi: P_{i} & \stackrel{B}{\times} \operatorname{Gr}(D) \\
(p, V) & \mapsto \operatorname{Gr}(i) \times \operatorname{Gr}(D) \\
& \mapsto\left(p \mathbf{C}^{i}, p V\right)
\end{aligned}
$$

takes $\psi\left(s_{i}, z_{D}\right)=z_{\Lambda_{i} D}$, the $B$-generating point of $\mathcal{F}_{\Lambda_{i} D}^{B}$. Thus $\psi: P_{i} \times \mathcal{F}_{D}^{B} \rightarrow \mathcal{F}_{\Lambda_{i} D}^{B}$ is an isomorphism.

(ii+iii) By the above, the projection is a bijection on the open B-orbit, and hence is birational. The image of the projection is $P_{i} \cdot \mathcal{F}_{D}^{B}$, which must be closed since $P_{i} \times{ }^{B} \mathcal{F}_{D}^{B}$ is a proper (i.e. compact variety).

(iv) Clear from the definitions.

Lemma 12. Let $(D, \mathbf{m})$ be a strongly separated family and $i \in[n-1]$. Let

$$
\begin{aligned}
& \mathcal{F}^{\prime}=P_{i} \stackrel{B}{\times} \mathcal{F}_{D}^{B} \\
& \mathcal{L}^{\prime}=P_{i} \stackrel{B}{\times} \mathcal{L}_{\mathbf{m}} .
\end{aligned}
$$

so that $\mathcal{L}^{\prime} \rightarrow \mathcal{F}^{\prime}$ is a line bundle. Then

$$
\operatorname{char}^{*} H^{0}\left(\mathcal{F}^{\prime}, \mathcal{L}^{\prime}\right)=\Lambda_{i} \operatorname{char}^{*} H^{0}\left(\mathcal{F}_{D}^{B}, \mathcal{L}_{\mathbf{m}}\right) .
$$


Proof. By Demazure's analysis of induction to $P_{i}$ (see [7], "construction élémentaire") we have

$$
\Lambda_{i} \operatorname{char}^{*} H^{0}\left(\mathcal{F}_{D}^{B}, \mathcal{L}_{\mathbf{m}}\right)=\operatorname{char}^{*} H^{0}\left(\mathcal{F}^{\prime}, \mathcal{L}^{\prime}\right)-\operatorname{char}^{*} H^{1}\left(P_{i} / B, H^{1}\left(\mathcal{F}_{D}^{B}, \mathcal{L}_{\mathbf{m}}\right)\right)
$$

However, we know by [21], Prop. 28(a) that $H^{0}\left(\mathcal{F}_{D}^{B}, \mathcal{L}_{\mathbf{m}}\right)$ has a good filtration, so that the $H^{1}$ term above is zero.

Corollary 13. If $(D, \mathbf{m})$ is strongly separated and $i$-free, and $\left(\Lambda_{i} D, \widetilde{\mathbf{m}}\right)$ is a diagram with multiplicities $\widetilde{\mathbf{m}}\left(s_{i} C\right)=m(C)$ for $C \in D, \widetilde{\mathbf{m}}\left(s_{i}[i]\right)=m_{0}$, then

$$
\operatorname{char}^{*} M_{\Lambda_{i} D, \widetilde{\mathbf{m}}}^{B}=\Lambda_{i} \varpi_{i}^{m_{0}} \operatorname{char}^{*} M_{D, \mathbf{m}}^{B} \text {. }
$$

If $m_{0}=0$, then

$$
\operatorname{char}^{*} M_{s_{i} D, \mathbf{m}}^{B}=\operatorname{char}^{*} M_{\Lambda_{i} D, \tilde{\mathbf{m}}}^{B}=\Lambda_{i} \operatorname{char}^{*} M_{D, \mathbf{m}}^{B}
$$

This follows immediately from the above Lemmas and Proposition 9.

Proof of Proposition. The first formula of the Proposition now follows from the above Lemmas and Prop. 9. The second statement follows similarly from Demazure's character formula and the vanishing statements of [21], Prop. 28.

\section{Schubert polynomials}

We now apply our theory to compute the Schubert polynomials $\mathcal{S}(w)$ of permutations $w \in S_{n}$, which generalize the Schur polynomials $s_{\lambda}\left(x_{1}, \ldots, x_{k}\right)$. They were originally considered as representatives of Schubert classes in the Borel picture of the cohomology of the flag variety $G L(n) / B$, though we will give a completely different geometric interpretation in $\S 4.2$. As a general reference, see Macdonald [20] or Fulton [10].

Although our results follow from the geometric theory of previous sections, we phrase them in a purely elementary and self-contained way (except in $\S 4.2$ ). Most of our computations in $\S \S 4.3-4.5$ are valid for the character of the generalized Schur module of any strongly separated family.

We first state the combinatorial definition of Schubert polynomials, and then prove the theorem of Kraskiewicz and Pragacz [12], that Schubert modules are the characters of flagged Schur modules associated to a Rothe diagram. Finally, we give three new, explicit formulas for Schubert polynomials. 


\subsection{Definitions}

The Schubert polynomials $\mathcal{S}(w)$ in variables $x_{1} \ldots, x_{n}$ are constructed combinatorially in terms of the following divided difference operators. First, the operator $\partial_{i}$ is defined by

$$
\partial_{i} f\left(x_{1}, \ldots, x_{n}\right)=\frac{f\left(x_{1}, \ldots, x_{i}, x_{i+1}, \ldots, x_{n}\right)-f\left(x_{1}, \ldots, x_{i+1}, x_{i}, \ldots, x_{n}\right)}{x_{i}-x_{i+1}} .
$$

Then for a reduced decomposition of a permutation $u=s_{i_{1}} s_{i_{2}} \cdots$, the operator $\partial_{u}=\partial_{i_{1}} \partial_{i_{2}} \ldots$ is independent of the reduced decomposition chosen. Also, take $\partial_{e}=\mathrm{id}$.

Now we may define the Schubert polynomials as follows. Let $w_{0}$ be the longest permutation $\left(w_{0}(i)=n+1-i\right)$, and take $u=w^{-1} w_{0}$, so that $w u=w_{0}$. Then

$$
\mathcal{S}(w) \stackrel{\text { def }}{=} \partial_{u}\left(x_{1}^{n-1} x_{2}^{n-2} \cdots x_{n-2}^{2} x_{n-1}\right) .
$$

We have $\operatorname{deg} \mathcal{S}(w)=\ell(w)$.

To compute any $\mathcal{S}(w)$, we write $w_{0}=w s_{i_{1}} \cdots s_{i_{r}}$ for some reduced word $s_{i_{1}} \cdots s_{i_{r}}\left(s_{i}=(i, i+1)\right.$ denoting a simple transposition in $\left.S_{n}\right)$. In particular, we may take $i_{k}$ to be the first ascent of $w_{k}=w s_{i_{1}} \cdots s_{i_{k-1}}$; that is, $i_{k}=$ the smallest $i$ such that $w_{k}(i+1)>w_{k}(i)$.

Examples. (a) For $w \in S_{3}$, we have $\mathcal{S}\left(w_{0}\right)=x_{1}^{2} x_{2}, \mathcal{S}\left(s_{1} s_{2}\right)=x_{1} x_{2}, \mathcal{S}\left(s_{2} s_{1}\right)=$ $x_{1}^{2}, \mathcal{S}\left(s_{2}\right)=x_{1}+x_{2}, \mathcal{S}\left(s_{1}\right)=x_{1}, \mathcal{S}(e)=1$.

(b) For the permutation $w=24153 \in S_{5}$, by inverting first ascents we get $w s_{1} s_{3} s_{2} s_{1} s_{4} s_{3}=w_{0}$, so

$$
\begin{aligned}
\mathcal{S}(w) & =\partial_{1} \partial_{3} \partial_{2} \partial_{1} \partial_{4} \partial_{3}\left(x_{1}^{4} x_{2}^{3} x_{3}^{2} x_{4}\right) \\
& =x_{1} x_{2}\left(x_{1} x_{2}+x_{1} x_{3}+x_{2} x_{3}+x_{1} x_{4}+x_{2} x_{4}\right) .
\end{aligned}
$$

(c) Given $n>k$, the partition $\lambda=\left(0 \leq \lambda_{1} \leq \lambda_{2} \leq \cdots \lambda_{k} \leq n\right)$ is "strictified" to the subset $C=\left\{\lambda_{1}+1<\lambda_{2}+2<\cdots \lambda_{k}+k\right\} \subset[n]$, which is completed to a Grassmannian permutation $w$ by adjoining $[n] \backslash C$. Then the Schubert polynomial of $w$ is equal to the Schur polynomial of $\lambda: \mathcal{S}(w)=s_{\lambda}\left(x_{1}, \ldots, x_{k}\right)$. For instance, for $n=7, k=5, C=12457, \lambda=00112$, we have $\mathcal{S}(1245736)=s_{00112}\left(x_{1}, \ldots, x_{5}\right)$.

Now, a diagram (generalized Young diagram) is a subset $D \subset \mathbf{N} \times \mathbf{N}$. The point $(i, j)$ is in row $i$, column $j$, and we think of a diagram as a list of columns $C \subset \mathbf{N}: D=\left(C_{1}, C_{2}, \ldots\right)$. Two diagrams are column equivalent, $D \cong D^{\prime}$, if one is obtained from the other by switching the order of columns (and ignoring empty columns). For a column $C \subset \mathbf{N}$, the multiplicity mult $_{D}(C)$ is the number of columns of $D$ with content equal to $C$. An equivalence class of diagrams is another way to express our subset families with multiplicity in $\S 3.1$. Sum of diagrams

$$
D \oplus D^{\prime}
$$


means placing $D$ horizontally next to $D^{\prime}$ (concatenating lists of columns), and $D \backslash\{C\}$ means removing one column whose content is equal to $C$.

The Rothe diagram of a permutation $w \in S_{n}$ is

$$
D(w)=\left\{(i, j) \in[n] \times[n] \mid i<w^{-1}(j), j<w(i)\right\} .
$$

It is easy to see that $D(w)$ is a strongly separated subset family. (In fact, it is northwest. See [23], [24], [21].)

Example. For the same $w=24153$, we have

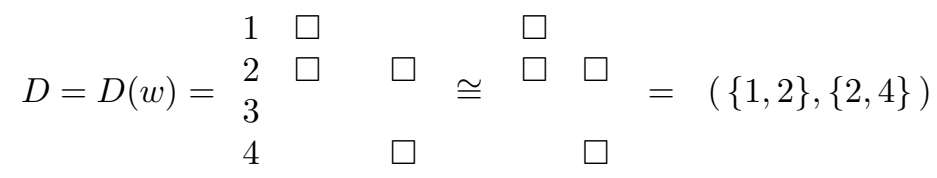

Recall that $[i]$ denotes the interval $\{1,2,3, \ldots, i\}$.

\subsection{Theorem of Kraskiewicz and Pragacz}

The geometric significance of the Schubert polynomials is as follows. There are two classical computations of the singular cohomology ring $H^{*}(G / B, \mathbf{C})$ of the flag variety. That of Borel identifies the cohomology with a coinvariant algebra

$$
c: H^{\cdot}(G / B, \mathbf{C}) \stackrel{\sim}{\rightarrow} \mathbf{C}\left[x_{1}, \ldots, x_{n}\right] / I_{+},
$$

where $I_{+}$is the the ideal generated by the non-constant symmetric polynomials. The map $c$ is an isomorphism of graded $\mathbf{C}$-algebras, and the generator $x_{i}$ represents the Chern class of the $i^{\text {th }}$ quotient of the tautological flag bundle, which is not the dual of an effective divisor. The alternative computation of Schubert gives as a linear basis for $H^{\cdot}(G / B, \mathbf{C})$ the Schubert classes $\sigma_{w}=\left[X_{w_{0} w}\right]$, the Poincare duals of the Schubert varieties.

The isomorphism between these computations was defined by Bernstein-GelfandGelfand [4] and Demazure [7], and given a precise combinatorial form by Lascoux and Schutzenberger [15]. It states that the Schubert polynomials $\mathcal{S}(w)$ defined above are representatives of the Schubert classes in the cohomology ring.

We now give a completely different geometric interpretation of the polynomials $\mathcal{S}(w)$ in terms of Weyl modules.

Theorem 14. (Kraskiewicz-Pragacz [12])

$$
\mathcal{S}(w)=\operatorname{char}^{*} M_{D(w)}^{B},
$$

where $M_{D(w)}^{B}$ is the Weyl module of $\S 3.1$ associated to $D(w)$ (thought of as a subset family with multiplicity). 
Proof. (Magyar-Reiner-Shimozono) Let $\chi(w)=\operatorname{char}^{*} M_{D(w)}^{B}$. We must show that $\chi(w)$ satisfies the defining relations of $\mathcal{S}(w)$.

First, $D\left(w_{0}\right)=([1], \ldots,[n-1])$, and

$$
M_{D\left(w_{0}\right)}^{B}=\mathbf{C} \cdot \widetilde{\Delta}_{1}^{1} \widetilde{\Delta}_{12}^{12} \ldots \widetilde{\Delta}_{[n-1]}^{[n-1]},
$$

a one-dimensional $B$-module, so $\chi\left(w_{0}\right)=x_{1}^{n-1} x_{2}^{n-2} \cdots x_{n-1}$.

Now, suppose $w s_{i}<w$, and $i$ is the first ascent of $w s_{i}$. Then the $w(i+1)^{\text {th }}$ element of $D(w)$ is $C_{w(i+1)}(w)=[i]$. Letting

$$
D^{\prime}(w) \stackrel{\text { def }}{=} D(w) \backslash\{[i]\}
$$

it is easily seen that:

(i) $D^{\prime}(w)$ is $i$-free,

(ii) $D(w) \cong D^{\prime}(w) \oplus\{[i]\}$, and

(iii) $D\left(w s_{i}\right) \cong s_{i} D^{\prime}(w) \oplus\{[i-1]\}$ (where $\left.[0]=\emptyset\right)$.

Hence we obtain trivially:

$$
\begin{aligned}
\chi(w) & =x_{1} \cdots x_{i} \operatorname{char}^{*} M_{D^{\prime}(w)}^{B} \\
\chi\left(w s_{i}\right) & =x_{1} \cdots x_{i-1} \operatorname{char}^{*} M_{s_{i} D^{\prime}(w)}^{B} .
\end{aligned}
$$

Since $D^{\prime}(w)$ is strongly separated and $i$-free, Corollary 13 implies that

$$
\operatorname{char}^{*} M_{s_{i} D^{\prime}(w)}^{B}=\Lambda_{i} \operatorname{char}^{*} M_{D^{\prime}(w)}^{B} .
$$

Thus we have

$$
\begin{aligned}
\chi\left(w s_{i}\right) & =\left(x_{1} \cdots x_{i-1}\right) \Lambda_{i} \operatorname{char}^{*} M_{D^{\prime}(w)}^{B} \\
& =\Lambda_{i} x_{i}^{-1}\left(x_{1} \cdots x_{i}\right) \operatorname{char}^{*} M_{D^{\prime}(w)}^{B} \\
& =\Lambda_{i} x_{i}^{-1} \chi(w) \\
& =\partial_{i} \chi(w) .
\end{aligned}
$$

But now, using the the first-ascent sequence to write $w_{0}=w s_{i_{1}} \cdots s_{i_{r}}$, we compute

$$
\chi(w)=\partial_{i_{1}} \cdots \partial_{i_{r}}\left(x_{1}^{n-1} x_{2}^{n-2} \cdots x_{n-1}\right)=\mathcal{S}(w) .
$$




\subsection{Orthodontia and Demazure character formula}

We will use the Demazure character formula (Prop. 10) to compute Schubert polynomials. To make this formula explicit, however, we must embed our Rothe diagram into a chamber family. The algorithm we give below will work for any strongly separated family.

Let $D=\left(C_{1}, C_{2}, \ldots\right)$ be a Rothe diagram. We require a reduced word $\mathbf{i}=$ $\left(i_{1}, \ldots, i_{l}\right)$ and a multiplicity list $\mathbf{m}=\left(k_{1}, \ldots, k_{n}, m_{1}, \ldots, m_{l}\right), k_{i}, m_{j} \geq 0$, which generate $D$ in the following sense. Define a diagram by

$$
D_{\mathbf{i}, \mathbf{m}}=\bigoplus_{i=1}^{n} k_{i} \cdot[i] \quad \oplus \bigoplus_{j=1}^{l} m_{j} \cdot\left(s_{i_{1}} s_{i_{2}} \cdots s_{i_{j}}\left[i_{j}\right]\right),
$$

where $m \cdot C=C \oplus \cdots \oplus C(m$ copies of $C)$, and $0 \cdot C=\emptyset$, an empty column. Then we require that $D \cong D_{\mathbf{i}, \mathbf{m}}$.

As our first step in generating $\mathbf{i}$ and $\mathbf{m}$, let $k_{i}=\operatorname{mult}_{D}([i]), 1 \leq i \leq n$, and remove from $D$ all columns of the form $C=[i]$ to get a new diagram $D_{-}$.

Given a column $C \subset[n]$, a missing tooth of $C$ is a positive integer $i$ such that $i \notin C$, but $i+1 \in C$. The only $C$ without any missing teeth are the intervals $[i]$, so we can choose a missing tooth $i_{1}$ of the first column of $D_{-}$. Now switch rows $i_{1}$ and $i_{1}+1$ of $D_{-}=\left\{C_{1}, C_{2}, \ldots\right\}$ to get a new diagram $D^{\prime}$ with closer teeth (orthodontia). That is,

$$
D^{\prime}=s_{i_{1}} D_{-}=\left\{s_{i_{1}} C_{1}, s_{i_{1}} C_{2}, \ldots\right\} .
$$

In the second step, repeat the above with $D^{\prime}$ instead of $D$. That is, let $m_{1}=$ mult $_{D^{\prime}}\left(\left[i_{1}\right]\right)$, and remove all columns of the form $C=\left[i_{1}\right]$ from $D^{\prime}$ to get a new diagram $D_{-}^{\prime}$. Find a missing tooth $i_{2}$ of the first column of $D_{-}^{\prime}$, and switch rows to get a new diagram $D^{\prime \prime}=s_{i_{2}} D_{-}^{\prime}$.

Iterate this procedure until all columns have been removed. It is easily seen that the sequences $\mathbf{i}$ and $\mathbf{m}$ thus defined have the desired properties.

Example. For $w=24153$,

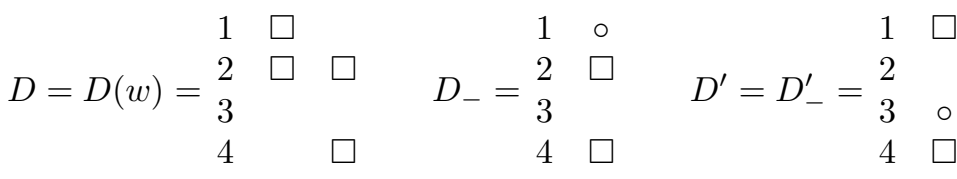

$$
\begin{aligned}
& D^{\prime \prime}=D_{-}^{\prime \prime}=\begin{array}{cc}
1 & \square \\
2 & \circ \\
3 & \square
\end{array} \quad D^{\prime \prime \prime}=\begin{array}{cc}
1 & \square \\
2 & \square
\end{array} \quad D_{-}^{\prime \prime \prime}=\emptyset
\end{aligned}
$$

so that the sequence of missing teeth (as indicated by $\circ$ ) gives $\mathbf{i}=(1,3,2)$, and $\mathbf{m}=\left(k_{1}=0, k_{2}=1, k_{3}=0, k_{4}=0, m_{1}=0, m_{2}=0, m_{3}=1\right)$. Furthermore $D=\left\{[2]^{1},\left(s_{1} s_{3} s_{2}[2]\right)^{1}\right\}=\{\{1,2\},\{2,4\}\}$. 
Note that $s_{i_{1}} \cdots s_{i_{l}}=s_{1} s_{3} s_{2}$ is a reduced subword of the first-ascent sequence $s_{1} s_{3} s_{2} s_{1} s_{4} s_{3}$ which raises $w$ to the maximal permutation $w_{0}$. This is always the case, and we can give an algorithm for extracting this subword.

Note. To apply this algorithm to a general strongly separated family $D$ (with multiplicity), first choose an order $D=\left\{C_{1}, C_{2}, \ldots\right\}$ for the subsets in the family (the columns) such that if $i<j$ then $\left(C_{i} \backslash C_{j}\right) \stackrel{\text { dt }}{<}\left(C_{j} \backslash C_{i}\right)$, in the notation of $\S 2.2$. For example, the obvious lexicographic order will do.

Now, the definition of $\mathcal{S}(w)$ involves descending induction (lowering the degree), but we give the following ascending algorithm, which follows immediately from Prop. 10.

Proposition 15. Given a permutation $w$, let $(\mathbf{i}, \mathbf{m})$ be a generating sequence such as the orthodontic sequence above. Let $\Lambda_{i}=\partial_{i} x_{i}$ and $\varpi_{i}=x_{1} x_{2} \cdots x_{i}$. Then

$$
\mathcal{S}(w)=\varpi_{1}^{k_{1}} \ldots \varpi_{n}^{k_{n}} \Lambda_{i_{1}}\left(\varpi_{i_{1}}^{m_{1}} \ldots\left(\Lambda_{i_{l}} \varpi_{i_{l}}^{m_{l}}\right) \ldots\right) .
$$

Example. For our permutation $w=24153$, we may verify that

$$
\mathcal{S}(w)=x_{1} x_{2} \Lambda_{1} \Lambda_{3} \Lambda_{2}\left(x_{1} x_{2}\right) .
$$

Note that this algorithm is more efficient than the usual one if the permutation $w \in S_{n}$ has small length compared to $n$.

Remark. The above proposition computes a Schubert polynomial $\mathcal{S}(w)$ in terms of a word $\mathbf{i}$. This word $\mathbf{i}$ is not a decomposition of $w$. We may view the formula of the proposition as computing the character of a space of sections over the BottSamelson variety $Z_{\mathbf{i}}$ (cf. $\S 3.3$ ). This variety is not the Schubert variety $X_{w}$, nor any desingularization of it, since in general $\operatorname{dim} X_{w} \neq \operatorname{dim} Z_{\mathbf{i}}$. There is no obvious combinatorial relationship between $w$ and $\mathbf{i}$, nor any obvious geometric relationship between $X_{w}$ and $Z_{\mathbf{i}}$.

\subsection{Young tableaux}

The work of Lascoux-Schutzenberger [17] and Littlemann [19] allows us to "quantize" our Demazure formula, realizing the terms of the polynomial by certain tableaux endowed with a crystal graph structure. Reiner and Shimozono have shown that our construction gives the same non-commutative Schubert polynomials as those in [16]. Our tableaux are different, however, from the "balanced tableaux" of Fomin, Greene, Reiner, and Shimozono [11]. For proofs see [14], and see also [24], [25]. 
Recall that a column-strict filling (with entries in $\{1, \ldots, n\}$ ) of a diagram $D$ is a map $t$, mapping the points $(i, j)$ of $D$ to numbers from 1 to $n$, strictly increasing down each column. The weight of a filling $t$ is the monomial $x^{t}=\prod_{(i, j) \in D} x_{t(i, j)}$, so that the exponent of $x_{i}$ is the number of times $i$ appears in the filling. We will define a set of fillings $\mathcal{T}$ of the Rothe diagram $D(w)$ which satisfy

$$
\mathcal{S}(w)=\sum_{t \in \mathcal{T}} x^{t} .
$$

We will need the root operators first defined in [17]. These are operators $f_{i}$ which take a filling $t$ of a diagram $D$ either to another filling of $D$ or are undefined. To define them we first encode a filling $t$ in terms of its reading word: that is, the sequence of its entries starting at the upper left corner, and reading down the columns one after another: $t(1,1), t(2,1), t(3,1), \ldots, t(1,2), t(2,2), \ldots$.

If it is defined, the lowering operator $f_{i}$ changes one of the $i$ entries to $i+1$, according to the following rule. First, we ignore all the entries in $t$ except those containing $i$ or $i+1$; if an $i$ is followed by an $i+1$ (ignoring non $i$ or $i+1$ entries in between), then henceforth we ignore that pair of entries; we look again for an $i$ followed (up to ignored entries) by an $i+1$, and henceforth ignore this pair; and iterate until we obtain a subword of the form $i+1, i+1, \ldots, i+1, i, i, \ldots, i$. If there are no $i$ entries in this word, then $f_{i}(t)$ is undefined. If there are some $i$ entries, then the leftmost is changed to $i+1$.

For example, we apply $f_{2}$ to the word

$$
\begin{aligned}
& t=1222132142523 \\
& \text {. } 22 \text {. } 32 \text {. . } 2233 \\
& \text {. } 2 \text {. . } 2 \text {. . } 2 \text {. . } 3 \\
& \text {. } 2 . .2 . . .5 . \\
& f_{2}(t)=\begin{array}{llllllllllll}
1 & 3 & 2 & 1 & 3 & 2 & 1 & 4 & 2 & 2 & 3 & 3
\end{array} \\
& f_{2}^{2}(t)=\begin{array}{llllllllllll}
1 & 3 & 2 & 1 & 3 & 3 & 1 & 4 & 2 & 2 & 3 & 3
\end{array} \\
& f_{2}^{3}(t)=\text { undefined }
\end{aligned}
$$

Decoding the image word back into a filling of the same diagram $D$, we have defined our operators.

Moreover, define the quantized Demazure operator $\widetilde{\Lambda}_{i}$ taking a tableau $t$ to a set of tableaux:

$$
\widetilde{\Lambda}_{i}(t)=\left\{t, f_{i}(t),\left(f_{i}\right)^{2}(t), \ldots\right\}
$$

Also, for a set $\mathcal{T}$ of tableaux, $\widetilde{\Lambda}(\mathcal{T})=\bigcup_{t \in \mathcal{T}} \widetilde{\Lambda}(t)$. Note that this means ordinary union of sets, without counting any multiplicities.

Now, consider the column $\phi_{i}=\{1,2, \ldots, i\}$ and its minimal column-strict filling $\varpi_{i}(j$ th row maps to $j)$. For a filling $t$ of any diagram $D=\left(C_{1}, C_{2}, \ldots\right)$, 
define in the obvious way the composite filling $\varpi_{i} \oplus t$ of the concatenated diagram $\phi_{i} \oplus D=\left(\phi_{i}, C_{1}, C_{2}, \ldots\right)$. This corresponds to concatenating the words $(1,2, \ldots, m)$ and $t$. Similarly, let $\left[\varpi_{i}\right]^{m} \oplus t$ denote concatenating $m$ copies of $\varpi_{m}$ before $t$.

Proposition 16. For a permutation $w$, let $\mathbf{i}, \mathbf{m}$ be a generating sequence as in the previous Proposition. Define the set of tableaux

$$
\mathcal{T}=\varpi_{1}^{k_{1}} \oplus \cdots \oplus \varpi_{n}^{k_{n}} \oplus \widetilde{\Lambda}_{i_{1}}\left(\varpi_{i_{1}}^{m_{1}} \oplus \ldots\left(\widetilde{\Lambda}_{i_{l}} \varpi_{i_{l}}^{m_{l}}\right) \ldots\right)
$$

Then the Schubert polynomial $\mathcal{S}(w)$ is the generating function of $\mathcal{T}$ :

$$
\mathcal{S}(w)=\sum_{t \in \mathcal{T}} x^{t}
$$

Proof. Follows immediately from the Demazure formula above, and the combinatorial properties of root operators described in [19] Sec. 5.

Example. Continuing the example of the previous section, the set $\mathcal{T}$ of tableaux (words) is built up as follows:

$$
\begin{gathered}
\left\{\varpi_{2}=12\right\} \stackrel{\widetilde{\Lambda}_{2}}{\longrightarrow}\{12,13\} \stackrel{\widetilde{\Lambda}_{3}}{\rightarrow}\{12,13,14\} \stackrel{\widetilde{\Lambda}_{1}}{\rightarrow}=\{12,13,14,23,24\} \\
\stackrel{\varpi_{2} \oplus}{\longrightarrow} \mathcal{T}=\mathcal{T}_{2}=\{1212,1213,1214,1223,1224\} .
\end{gathered}
$$

This clearly gives us the Schubert polynomial as generating function, and furthermore we see the crystal graph (with vertices the tableaux in $\mathcal{T}$ and edges all pairs of the form $\left.\left(t, f_{i} t\right)\right)$ :

$$
\begin{array}{cccc}
1223 & \stackrel{1}{\leftarrow} & 1213 & \\
3 \downarrow & & \downarrow & \\
1224 & \stackrel{1}{\leftarrow} & 1214 &
\end{array}
$$

The highest-weight elements in each component are the Yamanouchi words $\operatorname{Yam}(\mathcal{T})$ $=\{1213,1212\}$, and by looking at the corresponding lowest elements, we may deduce the expansion of the Schubert polynomial in terms of key polynomials (characters of Demazure modules): $\mathcal{S}(w)=\kappa_{x_{1} x_{2}^{2} x_{4}}+\kappa_{x_{1}^{2} x_{2}^{2}}=\kappa_{1201}+\kappa_{2200}$. Lascoux and Schutzenberger [17] have obtained another characterization of such lowest-weight tableaux. 


\subsection{Weyl character formula}

Our final formula reduces to the the Weyl character formula (Jacobi bialternant) in case $\mathcal{S}(w)$ is a Schur polynomial.

Geometrically, the idea is to apply the Atiyah-Bott Fixed Point Theorem to the Bott-Samelson variety to compute the character of its space of sections (the Schubert polynomial). This would be very inefficient, however, since the formula would involve $2^{l}$ terms (where $l$ is the length of the $\mathbf{i}$ found by orthodontia). We obtain a much smaller expression from considering a smaller configuration variety $\mathcal{F}_{\widetilde{D}}$ which is smooth and birational to the Bott-Samelson variety, and which desingularizes the configuration variety $\mathcal{F}_{D(w)}$. (See [21] for details.) The formula below applies also to the subset families of northwest type considered in [21], but for a general strongly separated family one has only the inefficient formula coming from the full Bott-Samelson resolution.

Combinatorially, we define a certain extension $\widetilde{D}$ of the Rothe diagram $D=$ $D(w)$. Define the staircase diagram to be the set of columns $\Phi=\{[1],[2], \ldots,[n]\}$. Let the flagged diagram $\Phi \oplus D$ be the sum (concatenation) of the two diagrams. Now, given $\Phi \oplus D=\left(C_{1}, \ldots, C_{r}\right)$, define the blowup of the flagged diagram $\widehat{\Phi \oplus D}=\left(C_{1}, \ldots, C_{r}, C_{1}^{\prime}, C_{2}^{\prime}, \ldots\right)$, where the extra columns are the intersections $\tilde{C}=C_{i_{1}} \cap C_{i_{2}} \cap \cdots \subset \mathbf{N}$, for all lists $C_{i_{1}}, C_{i_{2}}, \ldots$ of columns of $\Phi \oplus D$; but if an intersection $\tilde{C}$ is already a column of $\Phi \oplus D$, then we do not append it. Let $\widetilde{D}=\Phi \widehat{\oplus D}$.

Define a standard tabloid $t$ of $\widetilde{D}$ to be a column-strict filling such that if $C, C^{\prime}$ are columns of $\widetilde{D}$ with $C$ horizontally contained in $C^{\prime}$, then the numbers filling $C$ all appear in the filling of $C^{\prime}$. In symbols, $t: \widetilde{D} \rightarrow\{1, \ldots, n\}, t(i, j)<t(i+1, j)$ for all $i, j$, and $C \subset C^{\prime} \Rightarrow t(C) \subset t\left(C^{\prime}\right)$.

For $1 \leq i \neq j \leq n$ and a tabloid $t$ of $\widetilde{D}$, we define certain integers: $d_{i j}(t)$ is the number of connected components of the following graph. The vertices are columns $C$ of $\widetilde{D}$ such that $i \in t(C), j \notin t(C)$; the edges are $\left(C, C^{\prime}\right)$ such that $C \subset C^{\prime}$ or $C^{\prime} \subset C$.

Finally, since there are inclusions of diagrams $D, \Phi \subset \widetilde{D}$, we have the restrictions of a tabloid $t$ for $\widetilde{D}$ to $D$ and $\Phi$, which we denote $t \mid D$ and $t \mid \Phi$. For $t$ a filling of $\mathrm{D}$, let

$$
x^{t}=\prod_{(i, j) \in D} x_{t(i, j)}
$$

the weight of the filling.

\section{Proposition 17.}

$$
\mathcal{S}(w)=\sum_{t} \frac{x^{(t \mid D)}}{\prod_{i<j}\left(1-x_{i}^{-1} x_{j}\right)^{d_{i j}(t)-1}\left(1-x_{j}^{-1} x_{i}\right)^{d_{j i}(t)}},
$$

where $t$ runs over the standard tabloids for $\Phi \widehat{\oplus D}$ such that $(t \mid \Phi)(i, j)=i$ for all $(i, j) \in \Phi$. 
Example. For the same $w=24153$,

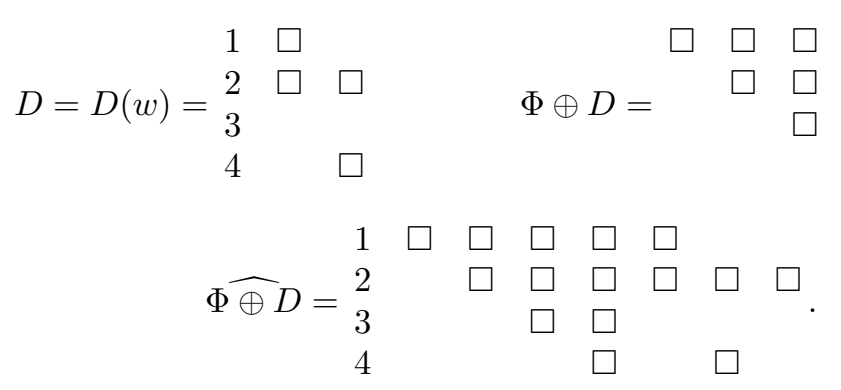

There are six standard tabloids of the type occurring in the theorem. Their restrictions to the last three columns of $\Phi \oplus D$ are:

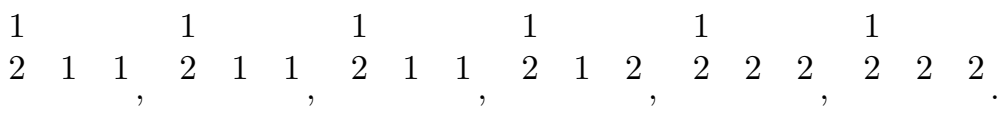

$$
\begin{aligned}
& \begin{array}{lllllll}
2 & 3 & 4 & 2 & 3 & 4
\end{array}
\end{aligned}
$$

The integers $d_{i j}(t)$ are 0,1 , or 2 , and we obtain

$$
\begin{aligned}
S(w)= & \frac{x_{1}^{2} x_{2}^{2}}{\left(1-x_{1}^{-1} x_{2}\right)\left(1-x_{2}^{-1} x_{3}\right)\left(1-x_{2}^{-1} x_{4}\right)}+\frac{x_{1}^{2} x_{2} x_{3}}{\left(1-x_{1}^{-1} x_{2}\right)\left(1-x_{3}^{-1} x_{4}\right)\left(1-x_{3}^{-1} x_{2}\right)} \\
+ & \frac{x_{1}^{2} x_{2} x_{4}}{\left(1-x_{1}^{-1} x_{2}\right)\left(1-x_{4}^{-1} x_{2}\right)\left(1-x_{4}^{-1} x_{3}\right)}+\frac{x_{1}^{2} x_{2}^{2}}{\left(1-x_{1}^{-1} x_{3}\right)\left(1-x_{1}^{-1} x_{4}\right)\left(1-x_{2}^{-1} x_{1}\right)} \\
& +\frac{x_{1} x_{2}^{2} x_{3}}{\left(1-x_{2}^{-1} x_{1}\right)\left(1-x_{3}^{-1} x_{4}\right)\left(1-x_{3}^{-1} x_{1}\right)}+\frac{x_{1} x_{2}^{2} x_{4}}{\left(1-x_{2}^{-1} x_{1}\right)\left(1-x_{4}^{-1} x_{1}\right)\left(1-x_{4}^{-1} x_{3}\right)} .
\end{aligned}
$$

Note that it is not clear a priori why this rational function should simplify to a polynomial (with positive integer coefficients).

\section{Acknowledgements}

The author would like to thank Bill Fulton, Victor Reiner, Mark Shimozono, and Andrei Zelevinsky for numerous helpful suggestions and for making available their unpublished work; as well as Wilberd van der Kallen for his essential help in pointing out the application of Mathieu's vanishing theorems in $\S 3.2$.

\section{References}

[1] K. Akin, D. Buchsbaum and J. Weyman, Schur functors and Schur complexes, Adv. Math. 44 (1982), 207-277.

[2] A. Berenstein, S. Fomin and A. Zelevinsky, Parametrizations of canonical bases and totally positive matrices, Adv. Math. 122 (1996), 49-149.

[3] A. Berenstein and A. Zelevinsky, Total positivity in Schubert varieties, Comment. Math. Helv. 72 (1997), 128-166. 
[4] I.N. Bernstein, I.M. Gelfand and S.I. Gelfand, Schubert cells and cohomology of the spaces G/P, Russ. Math. Surv. 28 (1973), 1-26.

[5] R. Bott and H. Samelson, Applications of the theory of Morse to symmetric spaces, J. Diff. Geom. 1 (1967), 311-330.

[6] N. Bourbaki, Groupes et Algèbres de Lie, Ch. 4, 5, et 6, Masson, Paris 1981.

[7] M. Demazure, Désingularisation des variétés de Schubert généralisés, Ann. Sci. Ec. Norm. Sup. 7 (1974), 53-88.

[8] M. Demazure, Une nouvelle formule des caractères, Bull. Sci. Math. (2) 98 (1974), 163-172.

[9] J. Desarmenien, J.P.S. Kung and G.-C. Rota, Invariant theory, Young bitableaux, and combinatorics, Adv. Math. 27 (1978), 63-92.

[10] W. Fulton, Young Tableaux with Applications to Representation Theory and Geometry, Cambridge Univ. Press, 1996.

[11] S. Fomin, C. Greene, V. Reiner and M. Shimozono, Balanced labellings and Schubert polynomials, European J. Combin. 18 (1997), 373-389.

[12] W. Kraskiewicz and P. Pragacz, Foncteurs de Schubert, C.R. Acad. Sci. Paris 304 Ser I, 9 (1987), 207-211.

[13] V. Lakshmibai and P. Magyar, Standard monomial theory for Bott-Samelson varieties, C.R. Acad. Sci. Paris, Ser I, 324 (1997), 1211-1215.

[14] V. Lakshmibai and P. Magyar, Standard monomial theory for Bott-Samelson varieties of GL(n), preprint alg-geom/9703020, Publ. RIMS Kyoto 34 (1998), 229-248.

[15] A. Lascoux and M.-P. Schutzenberger, Polynomes de Schubert, C. R. Acad. Sci. Paris 294 (1982), 447-450.

[16] A. Lascoux and M.-P. Schutzenberger, Tableaux and non-commutative Schubert polynomials, Funkt. Anal. 23 (1989), 63-64.

[17] A. Lascoux and M.-P. Schutzenberger, Keys and standard bases, pp. 125-144. In: D. Stanton (Ed.), Tableaux and Invariant Theory, IMA, Math. and App. 19 (1990).

[18] B. Leclerc and A. Zelevinsky, Quasicommuting families of quantum Plucker coordinates, AMS Translation Series 181 (1998), 85-108.

[19] P. Littelmann, A Littlewood-Richardson rule for symmetrizable Kac-Moody algebras, Inv. Math. 116 (1994), 329-346.

[20] I.G. Macdonald, Notes on Schubert Polynomials, Pub. LCIM 6, Univ. du Québec a Montréal, 1991.

[21] P. Magyar, Borel-Weil theorem for Schur modules and configuration varieties, Adv. Math. 135 (1998), 328-366.

[22] O. Mathieu, Filtrations of G-modules, Ann. Sci. École Norm. Sup. 23 (1990), 625-644.

[23] V. Reiner and M. Shimozono, Key polynomials and a flagged Littlewood-Richardson rule, $J$. Comb. Th. Ser. A 70 (1995), 107-143.

[24] V. Reiner and M. Shimozono, Specht series for column-convex diagrams, J. Alg. 174 (1995), 489-522.

[25] V. Reiner and M. Shimozono, \%-Avoiding, Northwest shapes and peelable tableaux, preprint 1996.

[26] A. Zelevinsky, Small resolutions of singularities of Schubert varieties, Funct. Anal. App. 17 (1983), 142-144.

Peter Magyar

Department of Mathematics

Northeastern University

Boston, MA 02115, USA

e-mail: pmagyar@lynx.neu.edu

(Received: November 27, 1997) 